Draft Version June 22, 2021

Preprint typeset using ${ }^{A} \mathrm{TE}_{\mathrm{E}} \mathrm{X}$ style emulateapj v. 08/13/06

\title{
W40 REGION IN THE GOULD BELT : AN EMBEDDED CLUSTER AND H II REGION AT THE JUNCTION OF FILAMENTS
}

\author{
K. K. MALLiCK \\ Department of Astronomy and Astrophysics, Tata Institute of Fundamental Research, \\ Homi Bhabha Road, Colaba, Mumbai 400 005, India \\ M. S. N. KUMAR \\ Centro de Astrofísica da Universidade do Porto, Rua das Estrelas, \\ 4150-762 s/n Porto, Portugal \\ D. K. Олна \\ Department of Astronomy and Astrophysics, Tata Institute of Fundamental Research, \\ Homi Bhabha Road, Colaba, Mumbai 400 005, India \\ RAFAEL BACHILLER \\ Observatorio Astronmico Nacional (IGN), Alfonso XII 3, 28014, Madrid, Spain \\ M. R. SAMAL \\ Aix Marseille Université, CNRS, LAM (Laboratoire d'Astrophysique de Marseille) \\ UMR 7326, 13388 Marseille, France \\ AND \\ L. Pirogov \\ Institute of Applied Physics, Russian Academy of Sciences, \\ 46 Uljanov str., Nizhny Novgorod 603950, Russia \\ Draft version June 22, 2021
}

\section{ABSTRACT}

We present a multiwavelength study of the W40 star-forming region using infrared (IR) observations in UKIRT JHK bands, Spitzer IRAC bands, and Herschel PACS bands; $2.12 \mu \mathrm{m} \mathrm{H}_{2}$ narrow-band imaging; and radio continuum observations from GMRT (610 and $1280 \mathrm{MHz}$ ), in a field of view (FoV) of $\sim 34^{\prime} \times 40^{\prime}$. Archival Spitzer observations in conjunction with near-IR (NIR) observations are used to identify 1162 Class II/III and 40 Class I sources in the FoV. The nearest-neighbour stellar surface density analysis shows that majority of these young stellar objects (YSOs) constitute the embedded cluster centered on the high-mass source IRS 1A South. Some YSOs, predominantly younger population, are distributed along and trace the filamentary structures at lower stellar surface density. The cluster radius is obtained as $0.44 \mathrm{pc}$ - matching well with the extent of radio emission - with a peak density of $650 \mathrm{pc}^{-2}$. The $J H K$ data is used to map the extinction in the region which is subsequently used to compute the cloud mass. It has resulted in $126 \mathrm{M}_{\odot}$ and $71 \mathrm{M}_{\odot}$ for the central cluster and the northern IRS 5 region, respectively. $\mathrm{H}_{2}$ narrow-band imaging displays significant emission, which prominently resembles fluorescent emission arising at the borders of dense regions. Radio continuum analysis shows this region as having blister morphology, with the radio peak coinciding with a protostellar source. Free-free emission spectral energy distribution (SED) analysis is used to obtain physical parameters of the overall photoionized region and the IRS 5 sub-region. This multiwavelength scenario is suggestive of star formation having resulted from merging of multiple filaments to form a hub. Star formation seems to have taken place in two successive epochs, with the first epoch traced by the central cluster and the high-mass star(s) - followed by a second epoch which is spreading into the filaments as uncovered by the Class I sources and even younger protostellar sources along the filaments. The IRS $5 \mathrm{H}$ II region displays indications of swept-up material which has possibly led to the formation of protostars.

Subject headings: H II regions - ISM: bubbles - ISM: individual objects(W40) - Infrared: ISM - Radio continuum: ISM - Stars: formation

\section{INTRODUCTION}

W40 (Westerhout 1958) (also known as Sh2-64) is an optically visible $\mathrm{H}$ II region (Fich \& Blitz 1984) in the Serpens-Aquila Rift region. Located at $\alpha_{2000} \sim$

Electronic address: kshitiz@tifr.res.in $18^{h} 31^{m} 29^{s}, \delta_{2000} \sim-02^{\circ} 05^{\prime} 24^{\prime \prime}$, it is visible as a bipolar nebula at mid and far IR wavelengths; harbouring complex features such as filamentary structures and an embedded arc-shaped nebula (Figure 1).

Early CO observations of this region showed the W40 $\mathrm{H}$ II region to be located at the edge of the molecular 
cloud G28.8+3.5 (Zeilik \& Lada 1978), resulting in the well-known blister morphology. Even so, the full extent of the molecular gas in this region is not mapped so far. Only the central few arcminutes of this cloud are mapped, using isotopologues of $\mathrm{CO}$, finding the mass of central cloud core to be $\sim 100 M_{\odot}$ (Vallee et al. 1992). W40 also has an associated dense molecular clump of 20' diameter (TGU 279 P7; Dobashi et al. 2005). A large scale, weak molecular outflow is also found, through CO observations, to originate in the molecular cloud (Zhu et al. 2006). Located at a Galactic latitude of $\sim 3.5^{\circ}$, this region is above the main Galactic plane, and the distance estimates to it vary from 300 to 900 pc (Radhakrishnan et al. 1972; Shuping et al. 2012; Vallee 1987; Rodney \& Reipurth 2008, and references therein). In the present work, we have adopted a distance of 500 pc from Radhakrishnan et al. (1972); Shuping et al. (2012). The central region of the W40 molecular cloud and $\mathrm{H}$ II region is known to host an embedded cluster of young stars, with a significant population of early-type sources (Smith et al. 1985), with the earliest spectral type of about O9.5 (Shuping et al. 2012). This cluster of early-type sources also partially reveals itself as a cluster of compact radio sources, coexisting with other compact radio sources which are classified as candidate ultra-compact H II regions and radio variable sources (Rodríguez et al. 2010). Together, these observations show that the W40 molecular cloud/H II region is one of the few nearby regions with active star formation away from the Galactic plane, hosting an embedded cluster including high-mass stars, and thus representing a template laboratory to investigate the process of star formation.

Recent observations of the larger Serpens-Aquila Rift in the (sub)millimeter wavelengths include this region, and have produced a systematic, unbiased sample of starless as well as protostellar dense cores within this cloud (Bontemps et al. 2010; Maury et al. 2011). Also, high resolution X-ray observation using Chandra has revealed the near-complete census of young stellar population within the embedded cluster (Kuhn et al. 2010). Low frequency radio observations show newer compact sources in this region (Pirogov et al. 2012).

Despite the multitude of individual studies of the W40 molecular cloud, embedded cluster and $\mathrm{H}$ II region, at wavelengths ranging from $\mathrm{X}$-rays to radio, an analysis of the overall star formation scenario in W40 is pending. In this paper, the aim is to fill this lacuna by using the vast archival dataset together with new NIR and radio observations.

In Section 2, we present the observations (archival or otherwise) and data reduction procedures. We discuss the results pertaining to stellar sources (classification and their analysis), the morphology, and the physical parameters of the region from various datasets in Sections 3, 4 , and 5, respectively; followed by a discussion on the possible star formation scenario in Section 6. The main conclusions are presented in Section 7.

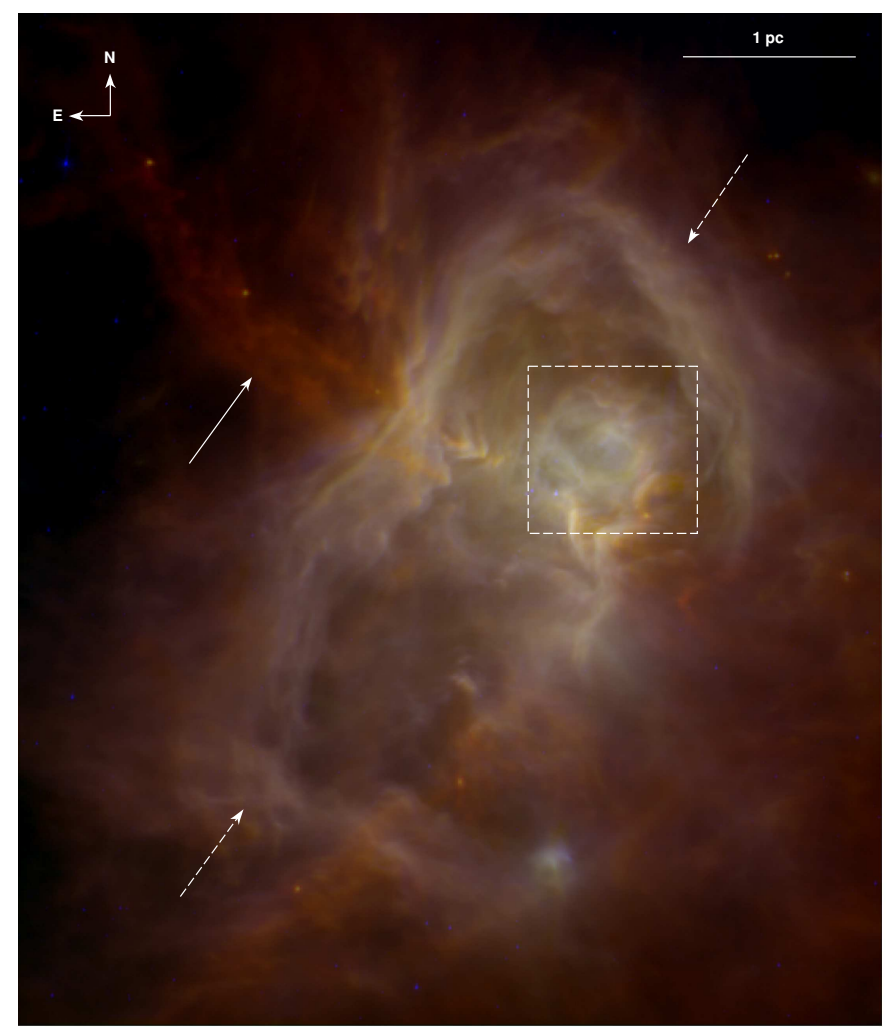

FIG. 1.- Colour composite image of the W40 region $\left(\sim 34^{\prime} \times 40^{\prime}\right)$ using Herschel PACS $160 \mu \mathrm{m}$ (red), $100 \mu \mathrm{m}$ (green), and IRAC 8 $\mu \mathrm{m}$ (blue) images. The dashed white arrows mark the two prominent northern and southern lobes of this bipolar nebula. The solid white arrow indicates one of the prominent filamentary structures. Dashed white box shows the location of a distinct smaller arcshaped nebula around IRS 5 source.

\section{OBSERVATIONS AND DATA ANALYSIS}

\subsection{Near Infrared Photometry}

NIR photometric imaging observations were carried out using the Wide Field Camera (WFCAM; Casali et al. 2007) mounted on the $3.8 \mathrm{~m}$ United Kingdom Infrared Telescope (UKIRT). WFCAM contains four Rockwell Hawaii-II (HgCdTe $2048 \times 2048$ pixel) arrays spaced by $94 \%$ in the focal plane. With a pixel scale of $0.4^{\prime \prime}$, the FoV of each array is $\sim 13.7^{\prime}$. JHK band data were obtained in the service mode during the period 2009 December 15-17. Observations at four positions are required to observe a contiguous $\left(\sim 0.75 \mathrm{deg}^{2}\right)$ square field - called a WFCAM 'tile'. Observation at each of these positions was taken using a nine point jitter pattern, and a $2 \times 2$ microstepping pattern, with an exposure time of $5 \mathrm{~s}$ each. The seeing was in the range $1.3^{\prime \prime}-1.8^{\prime \prime}$. We use the STARLINK software to do the initial processing, followed by mosaic making using the routine MAKEMOS in a manner similar to Davis et al. (2007) (see this paper for details of WFCAM processing).

The SExtractor software (Bertin \& Arnouts 1996) was used to detect all the stellar sources in the region. Source detection and deblending is highly effective with this software. A detection threshold of 3 , and a deblend contrast factor of 32 was used for our purposes. Subsequent to first pass of detection, the detection catalogue was filtered using the full-width-at-half-maxima (FWHM), stellarness parameter, and ellipticity to remove spurious de- 
tections. Finally, some leftover spurious detections were removed manually to create a reliable detection catalogue. Aperture photometry for the detected sources was performed using the PHOT routine in the IRAF APPHOT package. The aperture radius was set to 3 pixels (= $\left.1.2^{\prime \prime}\right)$, and the sky annulus was located 9 pixels away with a width of 7 pixels. Sources without any centering error (cier), sky fitting error (sier) and photometric error (pier) were retained for further work.

Instrumental magnitudes are in the WFCAM native system (the Mauna Kea Consortium Filter System or MKO system). Absolute photometric calibration of the $J, H$, and $K$ photometry was done using the Two Micron All Sky Survey (2MASS) stars in the FoV as explained below. We obtained 2MASS data with the constraints of "phqual = AAA" and "ccflag $=000 "$ for all the stars in the FoV. This ensures detections which have the highest photometric quality without neighbouring source confusion in all the three bands. We first constructed a $J$ $H / H-K$ colour-colour (CC) diagram using this data to identify only the main sequence (MS) stars in the region. The MS stars obtained using the CC diagram were then used to carry out the photometric calibration of the WFCAM data. The 2MASS data was converted to WFCAM system using the transformation equations given by Hodgkin et al. (2009). WFCAM data saturates for magnitudes brighter than $11.5 \mathrm{mag}$ in all the three bands, and these saturated points were replaced by $2 \mathrm{MASS}$ magnitudes.

Completeness limit tests were carried out for all the three bands by choosing subimages of size $5^{\prime} \times 5^{\prime}$ located in the cloud region and field region. The $90 \%$ completeness limits for the $J, H$, and $K$ band data are 19, 18, and $17 \mathrm{mag}$ - respectively - for the field region; and 18, 17, and 16 mag - respectively - for the cloud region; giving the mean as $18.5,17.5$, and $16.5 \mathrm{mag}$, respectively. These early observations of WFCAM have small differences in background matching between multiple chips and tiles, primarily resulting from the presence of large scale nebula contaminating the jittered frames. Therefore, the $J$, $H$, and $K$ catalogs were clipped at 18,17 and 16 mag limits for NIR CC, NIR colour-magnitude (CM), and extinction map analyses to obtain conservative and robust classifications of both young stellar objects (YSOs) and extinction measurements. Since all NIR magnitudes had low errors associated with them, they were not filtered by any error criteria. The magnitudes of bright sources which were saturated in the WFCAM image, but had good quality ("phqual=AAA") detections in 2MASS, were taken from the 2MASS catalogue after converting them to the WFCAM system using Hodgkin et al. (2009).

\subsection{Spitzer Photometry}

The Spitzer archival data for the Gould belt survey of Allen et al. (2006, PID : 30574) in all the four IRAC bands - 3.6 (Ch1), 4.5 (Ch2), 5.8 (Ch3), and 8.0 (Ch4) $\mu \mathrm{m}$ was obtained using the Spitzer Heritage Archive. Final image mosaics were created with the MOPEX data reduction software, by using the corrected basic calibrated data (cbcd) (S18.5 processed version), imask (bimsk), and uncertainty (cbunc) files. Mosaic images in all the four IRAC bands were created with a final pixel scale of $0.6^{\prime \prime}$.

Aperture photometry was carried out for the four
IRAC bands. We first used SExtractor for source detection as described above (see Section 2.1) and filtered to build a complete detection catalogue. The detection catalogue was used as input to the PHOT task in IRAF to obtain the aperture photometry of IRAC images. The aperture size, radius of the inner annulus, and radius of the outer annulus were taken as $2.4^{\prime \prime}, 2.4^{\prime \prime}$, and $7.2^{\prime \prime}$, respectively. The zero point magnitudes (with aperture corrections applied) were taken as 18.593, 18.090, 17.484, and 16.700 for the four IRAC bands, respectively; calculated using the IRAC Instrument Handbook (Version 2.0.2, March 2012).

Average completeness limits were determined using the peak of the luminosity function for the four bands. As is elucidated in the "Final Delivery Document For IRAC and MIPS data" 12 (see its Section 3.4.2), for c2d legacy project (Evans et al. 2003), the peak of the observed luminosity function can be assumed to estimate the $90 \%$ completeness limit. We constructed the luminosity functions with $0.5 \mathrm{mag}$ bin size for each band, and the peak (and thus the approximate $90 \%$ completeness limit) was found to be at $\sim 14,14,11.5$, and 11.5 for Ch1, Ch2, Ch3, and Ch4, respectively.

The south-east region of the wide-field image where the extinction is low was the only region where both WFCAM and Spitzer had a high density of sources causing source crowding problems. However, as mentioned in above sections, double-care was exercised in filtering first at the level of SExtractor and then at photometry allowing us to be quite certain that the detected sources were all real and uncontaminated. Finally, the cross-matching makes it even more robust.

\subsection{Cross-matching the Photometric Catalogues}

The number of source detections in individual photometric catalogues of each band are different due to respective band extinctions, point spread function (psf), sensitivity limits and area of coverage. Different crossmatched catalogues were produced for different analyses. The final analysis is done on an area of size $\sim 34.33^{\prime} \times$ $40.00^{\prime}$, centered at $\alpha_{2000} \sim 18^{h} 31^{m} 41.23^{s}, \delta_{2000} \sim$ $02^{\circ} 06^{\prime} 29.21^{\prime \prime}$. We obtained the following cross-matched and merged catalogues for various analyses :

- Ch1-Ch2-Ch3-Ch4 catalogue : The IRAC Ch1, Ch2, Ch3 and Ch4 band catalogues were crossmatched (within $0.6^{\prime \prime}$ ) with the constraint that all individual band errors are $\leq 0.15 \mathrm{mag}$ to produce an all-IRAC catalogue of 1874 sources. This was used in the classification of YSOs.

- H-K-Ch1-Ch2 catalogue : Not all sources are detected in IRAC Ch3 and Ch4 bands, but have good quality detections at $H$ and $K$ wavelengths. For classification of such sources into different evolutionary stages, we obtain a cross-matched catalogue of $H, K, \mathrm{Ch} 1$, and Ch2 magnitudes (NIR $H$ and $K$ catalogues were matched with $0.4^{\prime \prime}$ matching radius, Ch1 and Ch2 catalogues were matched with $0.6^{\prime \prime}$ matching radius; followed by matching the resultant NIR and Spitzer catalogues with $0.6^{\prime \prime}$

\footnotetext{
${ }^{1}$ http://peggysue.as.utexas.edu/SIRTF/

2 http://irsa.ipac.caltech.edu/data/SPITZER/C2D/
} 
radius) with good quality detections in IRAC Ch1 and $\mathrm{Ch} 2$ bands, i.e. sources with IRAC Ch1 and Ch2 magnitude errors $\leq 0.15$ mag. The resulting catalogue contains 8585 cross-matched sources.

- J-H-K catalogue : Next, we obtained a crossmatched (within $0.4^{\prime \prime}$ ) $J-H-K$ catalogue which was further clipped by conservative $J, H$, and $K$ completeness limits 18, 17, and $16 \mathrm{mag}$ as elucidated in Section 2.1. This filtered NIR catalogue containing 10990 sources was used for the identification of YSOs and to produce the extinction map (see Section 3.2) of the region.

- H-K catalogue : Many embedded NIR sources are detected only at $H$ and $K$ wavelengths. For identification of additional YSOs from amongst these, cross-matched NIR sources (within $0.4^{\prime \prime}$ ) with detections in only $H$ and $K$ bands (clipped at $H$ and $K$ conservative completeness limits of 17 and 16 , repectively) were obtained.

The above described catalogues are not mutually exclusive. Respective YSOs were identified using these in Section 3.1.

\section{4. $\mathrm{H}_{2}$ narrow-band imaging}

Narrow-band imaging in the $2.12 \mu \mathrm{m} \mathrm{H}_{2}(\mathrm{v}=1-0) \mathrm{S}(1)$ filter and broad band imaging in the $K^{\prime}$ for continuum subtraction was obtained for a $19^{\prime} \times 14^{\prime}$ region centered on the W40 embedded cluster. These data were obtained with the Calar-Alto $3.5 \mathrm{~m}$ telescope during the night of 2001 June 13 using the Omega Prime camera. The camera used an $\mathrm{HgCdTe}$ detector with $1024 \times 1024$ pixels with a plate scale of $0.39^{\prime \prime}$ pixel $^{-1}$ resulting in a $6.75^{\prime} \times 6.75^{\prime}$ FoV. Observations were carried out using a 9 point jitter pattern. The exposure time was $30 \mathrm{~s}$ and $3 \mathrm{~s}$ - per jitter pointing - in the NB2122 and $K^{\prime}$ filters respectively, resulting in total integration times of $180 \mathrm{~s}$ and $18 \mathrm{~s}$ per pixel, respectively. Standard image reduction of dark subtraction, flat-fielding and sky-subtraction was carried out for each pointing. The $K^{\prime}$ images were psf matched and scaled to the $\mathrm{H}_{2}$ narrow-band image to perform continuum subtraction.

\subsection{Herschel Archival Data}

Herschel Space Observatory, a $3.5 \mathrm{~m}$ telescope, was launched to carry out observations in the wavelength range 51-670 $\mu \mathrm{m}$ using the instruments Photodetector Array Camera and Spectrometer (PACS), SPIRE, and HIFI (Pilbratt et al. 2010). We obtained the publicly available level2_5 processed archival data for PACS 100 $\mu \mathrm{m}\left(3.2^{\prime \prime}\right.$ pixel $\left.^{-1}\right)$ and $160 \mu \mathrm{m}\left(6.4^{\prime \prime}\right.$ pixel $\left.^{-1}\right)$ bands - obtained as part of the Proposal ID:KGBT_pandre_1. The "MADmap" (Cantalupo et al. 2010; Waskett et al. 2007) processed products are used, as opposed to "PhotProject" processing, since our aim is to analyse extended features in the region. A detailed overview of procedures involved, data reduction pipeline, and data products is available on the Herschel website ${ }^{3} 4$ We use the images to examine the filamentary structures vis-a-vis the distribution of YSOs and radio continuum emission.

\footnotetext{
3 http://herschel.esac.esa.int/Data_Processing.shtml

4 http://herschel.esac.esa.int/Data_Products.shtml
}

TABLE 1

Details of GMRT OBservations

\begin{tabular}{ccc}
\hline \hline & $1280 \mathrm{MHz}$ & $610 \mathrm{MHz}$ \\
\hline Date of Obs. & $2011 \mathrm{Nov} .15$ & $2011 \mathrm{Nov} .18$ \\
Phase Center & $\alpha_{2000}=18^{h} 31^{m} 15.75^{s}$ & $\alpha_{2000}=18^{h} 31^{m} 15.75^{s}$ \\
& $\delta_{2000}=-02^{\circ} 06^{\prime} 49.30^{\prime \prime}$ & $\delta_{2000}=-02^{\circ} 06^{\prime} 49.30^{\prime \prime}$ \\
Flux Calibrator & $3 \mathrm{C} 286,3 \mathrm{C} 48$ & $3 \mathrm{C} 286,3 \mathrm{C} 48$ \\
Phase Calibrator & $1822-096$ & $1822-096$ \\
Cont. Bandwidth & $32 \mathrm{MHz}$ & $32 \mathrm{MHz}$ \\
Primary Beam & $24^{\prime}$ & $43^{\prime}$ \\
Resolution of maps & & $44.11^{\prime \prime} \times 40.67^{\prime \prime}$ \\
used for fitting & $45.84^{\prime \prime} \times 44.40^{\prime \prime}$ & $0.35 \mathrm{Jy} / \mathrm{beam}$ \\
Peak Flux Density & $0.45 \mathrm{Jy} / \mathrm{beam}$ & $5.52 \mathrm{~mJ} / \mathrm{beam}$ \\
rms noise & $6.34 \mathrm{~mJ} / \mathrm{beam}$ & \\
Total Integrated & & $4.07 \mathrm{Jy}$ \\
Flux Density & & \\
within 4 area & $7.55 \mathrm{Jy}$ & $121 \mathrm{mJy}$ \\
Integrated Flux & & \\
Density within & & \\
$50^{\prime \prime}$ radius of & & \\
IRS radio peak & $109 \mathrm{mJy}$ & \\
\hline
\end{tabular}

\subsection{Radio Continuum Observations}

New radio continuum observations are obtained using the Giant Metre-wave Radio Telescope (GMRT) in two bands centered at $1280 \mathrm{MHz}$ and $610 \mathrm{MHz}$. Data was obtained on 2011 November 15, and 2011 November 18 (Project ID 21_015), respectively, the details of which are given in Table 1. The GMRT array consists of 30 antennae located in an approximately $\mathrm{Y}$ shaped configuration. Each antenna has a diameter of $45 \mathrm{~m}$. A central region of about $1 \mathrm{~km} \times 1 \mathrm{~km}$ consists of 12 (randomly distributed) antennae, while the rest are distributed along three radial arms (6 along each arm) upto a distance of $\sim 14 \mathrm{~km}$. It results in a baseline of $\sim 100 \mathrm{~m}-25 \mathrm{~km}$, providing sensitivity to features $\lesssim 8^{\prime}$ and $17^{\prime}$ in the $1280 \mathrm{MHz}$ and $610 \mathrm{MHz}$ bands, respectively. Details about the GMRT array can be found in Swarup et al. (1991).

Data reduction was carried out with the AIPS software. Bad data were flagged using AIPS tasks "TVFLG" and "UVFLG". Multiple rounds of flagging and calibration were done to improve the calibration successively. Calibrated object data (W40) was subsequently split from the raw file (which contains phase and flux calibrator data also), following which low resolution $(\sim$ $\left.45^{\prime \prime} \times 45^{\prime \prime}\right)$ images were finally made using facet imaging with the help of the task "IMAGR". A few rounds of (phase) self-calibration were carried out to remove the ionospheric phase distortion effects.

\section{YOUNG STELLAR POPULATION \\ 3.1. Identification of $Y S O s$}

The YSOs were identified using sources detected in IRAC bands first, followed by using the catalogue of sources with detections in NIR and IRAC bands, and lastly using the NIR catalogue of sources. Following is an elucidation of the set of steps followed :

- Identification of YSOs from the IRAC data was accomplished by computing the IRAC spectral index for the sources with detections in all four IRAC bands (i.e sources from Ch1-Ch2-Ch3-Ch4 catalogue; see Section 2.3). The IRAC spectral index 

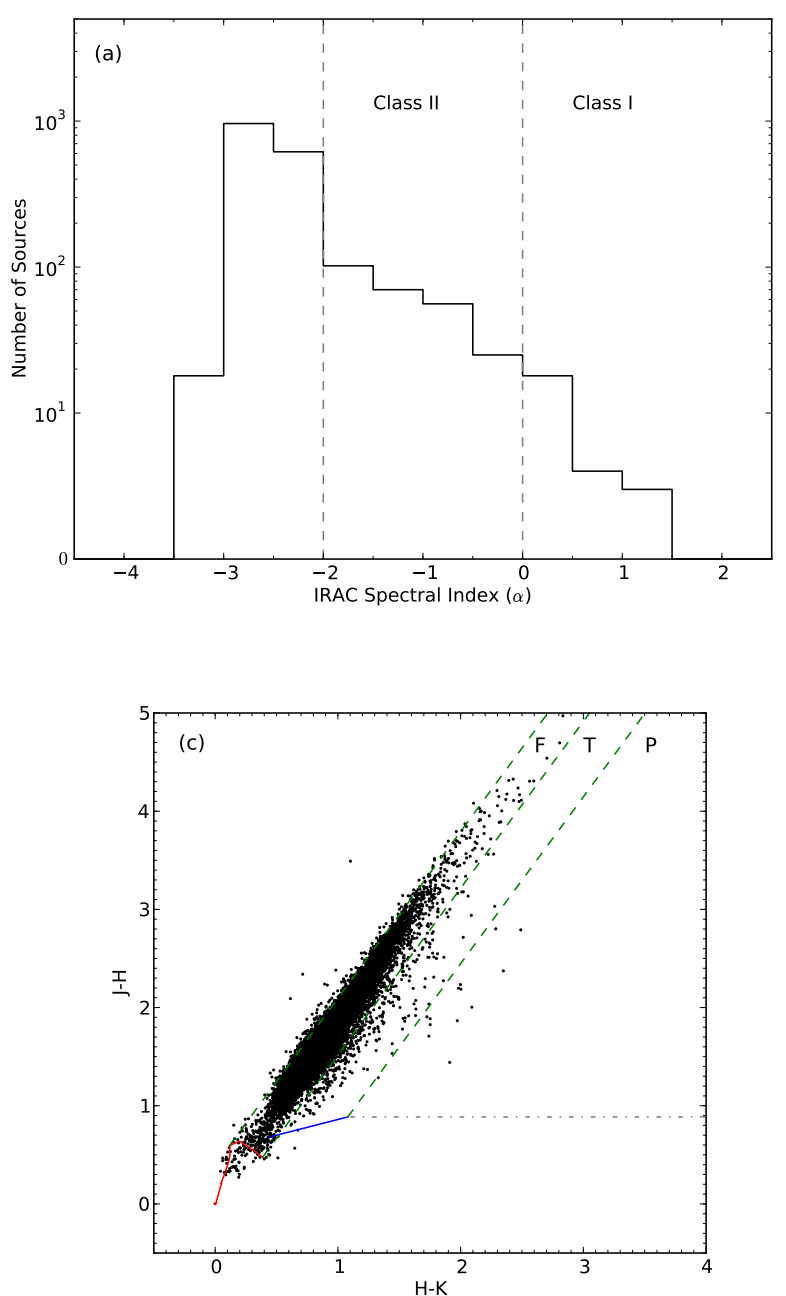
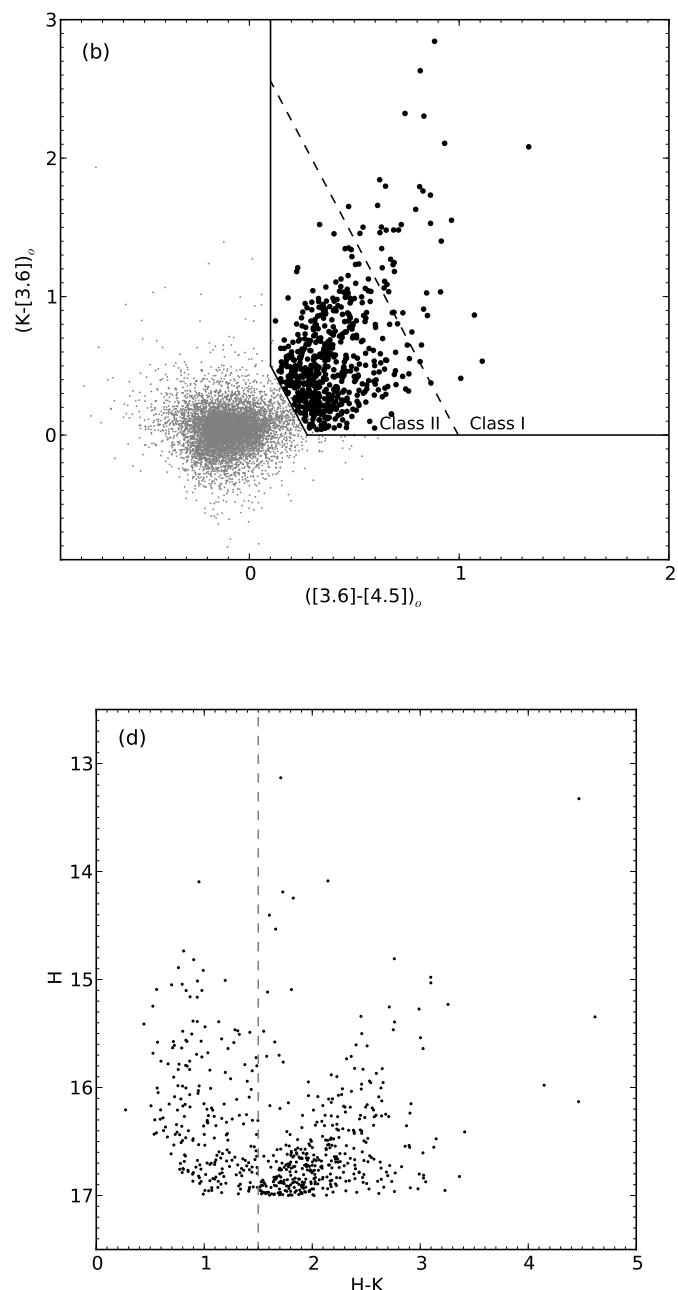

FIG. 2.- (a) Histogram of IRAC spectral index of sources. The boundary of Class I and Class II sources has been marked with vertical grey dashed lines. (b) NIR/IRAC CC plot showing the location of Class I and Class II sources, with areas demarcated (by black dashed and solid lines) using the procedure of Gutermuth et al. (2009). The filled black circles mark the sources identified. (c) $J-H / H-K$ CC diagram for the region. Solid blue line is the CTTS locus from Yasui et al. (2008). Red curve marks the locus of the dwarfs from Hewett et al. (2006). Dashed green lines are the reddening vectors, drawn (starting from the turning point, and the low-mass end of dwarf locus; and upper end of CTTS locus) using the reddening laws from Rieke \& Lebofsky (1985). Three regions - ' $\mathrm{F}$ ', ' $\mathrm{T}$ ', and 'P' mark the location of different classes of YSOs. The horizontal grey dot-dashed line has been drawn at $J-H=0.89$. All points are in MKO system. (d) $H / H-K$ CM diagram for the NIR sources with detections in only $H$ and $K$ bands. The grey vertical line $($ at $H-K=1.5)$ marks the cut-off upwards of which YSOs have been selected.

$\left(\alpha_{I R A C}=d \log \left(\lambda F_{\lambda}\right) / d \log (\lambda) ; \quad\right.$ Lada 1987), for each source, was calculated using linear regression. Thereafter, using the spectral index limits as given in Chavarría et al. (2008) (Class I : $0<\alpha_{I R A C}$, and Class II : $\left.-2 \leq \alpha_{I R A C} \leq 0\right)$, sources were classified into Class I and Class II categories (see Figure $2(\mathrm{a})$ ).

- H-K-Ch1-Ch2 cross-matched catalogue (see Section 2.3) was used for the identification of additional Class I and Class II sources using the classification scheme of Gutermuth et al. (2009, 2010, see their Appendix A - Phase 2). For this, the dereddened (K-[3.6]) and ([3.6]-[4.5]) colours, calculated using the colour excess ratios from Flaherty et al. (2007), were used. As is stated in Gutermuth et al. (2009), to filter out possible dim extragalactic contaminants, we also applied the limits on dereddened Ch1 band magnitude to be $\leq 15$ and $\leq 14.5$ for Class I and Class II categories, respectively. We used the average extinction value estimated in Section 3.2) (converted to $\mathrm{A}_{K}$ using Rieke \& Lebofsky 1985) to calculate the extinction for Ch1 band in this region, and thus intrinsic Ch1 magnitudes, to impose this condition. The reddening law from Flaherty et al. (2007) for Serpens (since it is closest to our region) was used for this purpose. Figure 2(b) shows the location of these Class I and Class II sources - which have been marked as filled black circles - on the $(K-[3.6])_{o}$ vs. $([3.6]-[4.5])_{o} \mathrm{CC}$ diagram.

- In the next step, the $J-H-K$ photometric catalogue was used as follows. For the sources in this catalogue, a $J-H / H-K$ CC diagram was constructed. Figure 2(c) shows the NIR $J-H / H-K$ CC diagram. The Classical T-Tauri Star (CTTS) locus (blue line) was taken from Yasui et al. (2008) (which is derived from Meyer et al. 1997), while the locus for dwarfs (red curve) in MKO system was taken from 
TABLE 2

YSOS IDENTIFIED IN THE FOV

\begin{tabular}{|c|c|c|c|c|c|c|c|c|}
\hline $\begin{array}{c}\mathrm{RA} \\
(\mathrm{J} 2000)\end{array}$ & $\begin{array}{c}\text { Dec } \\
(\mathrm{J} 2000)\end{array}$ & $\begin{array}{c}J \\
(\mathrm{mag})\end{array}$ & $\begin{array}{c}H \\
(\mathrm{mag})\end{array}$ & $\begin{array}{c}K \\
(\mathrm{mag})\end{array}$ & $\begin{array}{c}{[3.6]} \\
(\mathrm{mag})\end{array}$ & $\begin{array}{c}{[4.5]} \\
(\mathrm{mag})\end{array}$ & $\begin{array}{c}{[5.8]} \\
(\mathrm{mag})\end{array}$ & $\begin{array}{c}{[8.0]} \\
(\mathrm{mag})\end{array}$ \\
\hline 277.635620 & -2.305891 & $14.637 \pm 0.038$ & $11.474 \pm 0.025$ & $9.499 \pm 0.025$ & $8.417 \pm 0.002$ & $8.249 \pm 0.002$ & $7.810 \pm 0.004$ & $7.860 \pm 0.004$ \\
\hline 277.638031 & -2.118266 & $\ldots$ & $\ldots$ & $\ldots$ & $11.351 \pm 0.006$ & $10.719 \pm 0.006$ & $10.156 \pm 0.016$ & $9.422 \pm 0.016$ \\
\hline 277.638184 & -2.349572 & $14.582 \pm 0.048$ & $11.551 \pm 0.025$ & $9.273 \pm 0.020$ & $7.709 \pm 0.001$ & $6.973 \pm 0.001$ & $6.402 \pm 0.002$ & $6.179 \pm 0.001$ \\
\hline 277.638580 & -2.190570 & $\ldots$ & $\ldots$ & $\ldots$ & $10.309 \pm 0.004$ & $9.623 \pm 0.003$ & $8.998 \pm 0.009$ & $8.210 \pm 0.004$ \\
\hline 277.64144 .9 & -1.932326 & $\ldots$ & $14.189+0.002$ & $12.462+0.001$ & & & $\ldots$ & $\ldots$ \\
\hline
\end{tabular}

Hewett et al. (2006). Similar to Yasui et al. (2008), the reddening laws of Rieke \& Lebofsky (1985) $\left(\mathrm{A}_{J} / \mathrm{A}_{V}=0.282, \mathrm{~A}_{H} / \mathrm{A}_{V}=0.175\right.$, and $\mathrm{A}_{K} / \mathrm{A}_{V}=$ 0.112 ) were used. The dashed green lines show the reddening vectors. The sources which were located in the ' $\mathrm{T}$ ' and ' $\mathrm{P}$ ' regions - similar to the method of Ojha et al. (2004a,b), Lada \& Adams (1992) were taken as NIR Class II/III sources. There may be an overlap between the Herbig Ae/Be stars and Class II sources in this ' $\mathrm{T}$ ' region (Hillenbrand et al. 1992). To decrease any contamination in the ' $\mathrm{P}$ ' region, we took only those sources which were above $J-H=0.89$ threshold.

- Additionally, those NIR sources detected only in the $H$ and $K$ bands (i.e. using the $H$ - $K$ catalogue from Section 2.3) were also used to identify YSOs using the $H / H-K$ CM diagram (Figure $2(\mathrm{~d})$ ). From the $H / H-K \mathrm{CM}$ diagram, only the sources with an IR excess upwards of $H-K=1.5$ were selected. This cut-off was chosen as - in the CM diagram - it marks a clear low density gap between the field sources branch and the YSOs' branch. CM diagrams of nearby non-nebular regions also show the sources in those regions (i.e. the field sources) to be confined below this value. YSOs selected using the NIR photometry mostly fall into the Class II/III category.

The YSOs selected from each of the above steps are not mutually exclusive and there are overlapping YSOs between various methods. The selected YSOs were hence merged (with the class of a YSO taken as what it was identified as first in the above order of steps) to obtain a final catalog of 1202 YSOs, of which 40 are in the Class I category and 1162 in the Class II/III category. The central high-mass IRS sources (1A South, 2B, 3A, and 5) were detected as Class II in the above set of steps, but were expunged from the YSO catalogue as they have already been identified as main-sequence sources by Shuping et al. (2012). Table 2 gives the YSOs detected in the FoV. A sample of Table 2 is given here, whereas the complete table is available in electronic form as part of the online material.

\subsection{Extinction in the Region}

The NIR photometry from the new WFCAM data is deeper by two magnitudes compared to the 2MASS data. The NIR $J-H / H-K$ CC diagram of the region was used to select the reddened MS sources. Sources in the 'F' region (see Figure 2(c)), which were not classified as YSOs in Section 3.1, were selected for this analysis (a total of

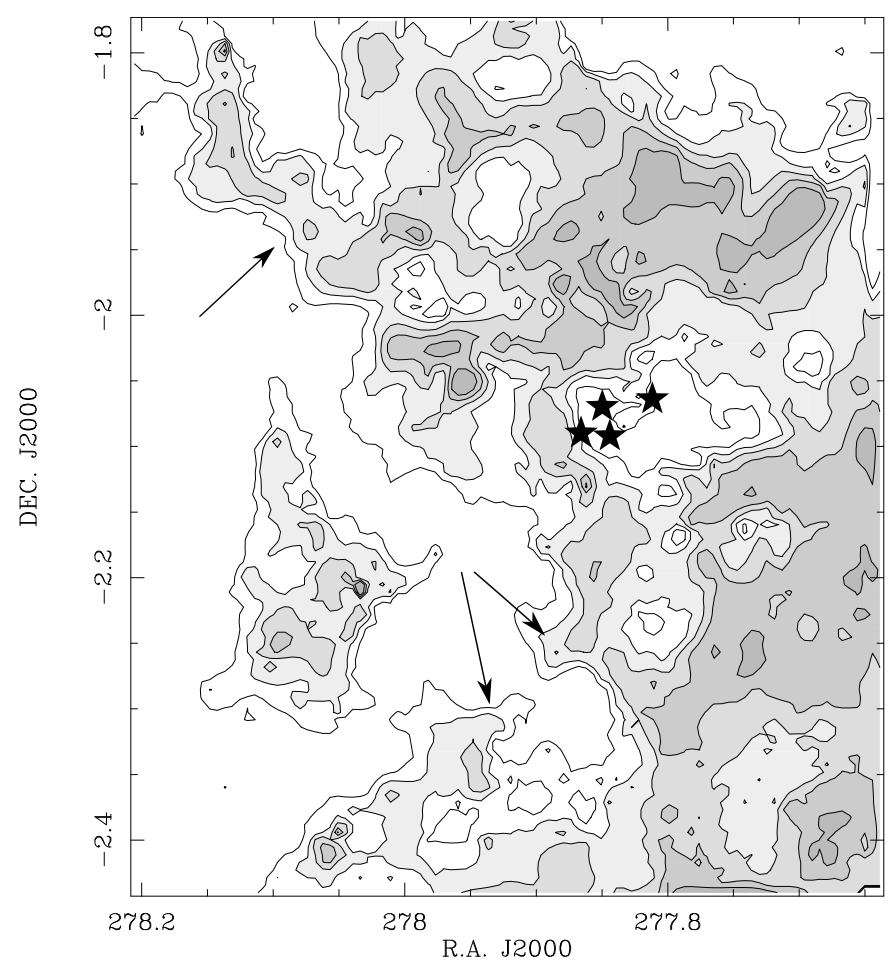

FIG. 3.- Visual extinction map of the region calculated using median value of 20 nearest neighbours. The $\left(\mathrm{A}_{V}\right)$ contours are drawn from 12 to $20 \mathrm{mag}$, with steps of 2 mag. The star symbols mark the location of central high-mass stars. Arrows indicate the locations of filamentary structures

10011 sources used here). We estimated the average visual extinction in the region by using a method similar to the Near-IR-Colour-Excess (NICE) method of Lada et al. (1994); Kainulainen et al. (2007); Ojha et al. (2004a). First, the $(H-K)$ colour excess for each of the selected sources was calculated by dereddening them, along the reddening vectors, to the dwarf locus which was approximated by a straight line asymptote. $A_{V}$, for each source, was subsequently calculated using the reddening laws of Rieke \& Lebofsky (1985).

The dereddenned extinction values were used to produce an extinction map of the region as shown in Figure 3. In producing this extinction map, at each position on the regular grid, a median extinction from the 20 valid nearest neighbours was computed. The choice of median instead of average value is because median works like an outlier rejection. Figure 3 displays two distinct features : a) a low extinction cavity around the ionizing stars, and b) higher extinction towards west and north. The filamentary structures (whose locations have been 


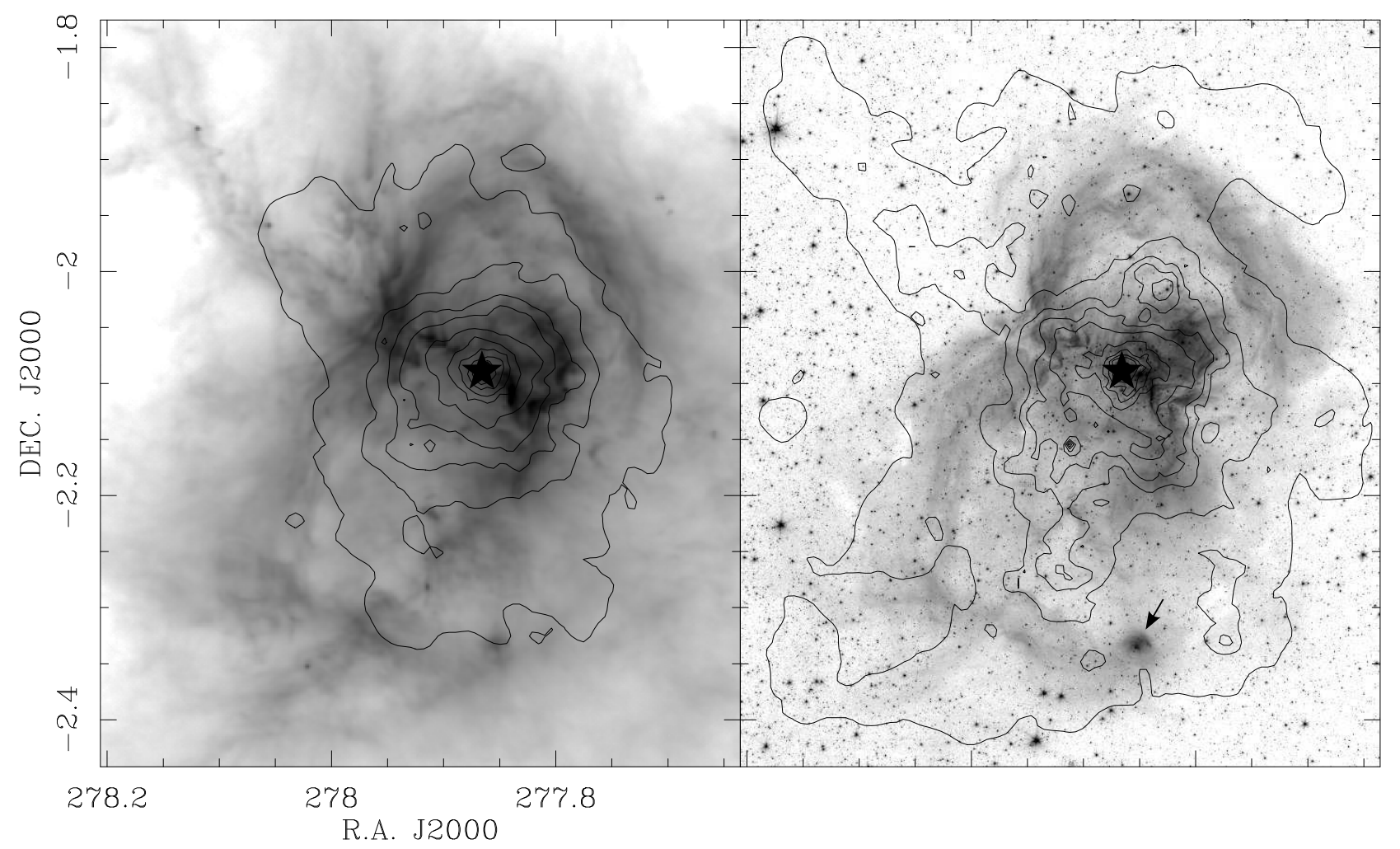

FIG. 4.- Surface Density contours of the region. (left) Made using 50 nearest neighbours and overlaid on PACS $160 \mu \mathrm{m}$ image. The contours are drawn at 40,70,100,130, 200,300,400,500, and 600 YSOs pc $^{-2}$. (right) Made using 20 nearest neighbours and overlaid on IRAC $3.6 \mu \mathrm{m}$ image. The contours are drawn at $25,50,75,100,150,275,400,525$, and $650 \mathrm{YSOs} \mathrm{pc}^{-2}$. The arrow marks a prominent compact nebula (see text). The star symbol in both the figures marks the location of IRS 1A.

marked with arrows) are partially traceable (with the one along north-east much more distinct than the one towards south). Although the filaments are found to be very dense from PACS $160 \mu \mathrm{m}$ emission (see Section 4.1), the extinction map fails to reproduce the same result because the NIR data is not deep enough to effectively detect many stars behind the filament. The depth of the NIR data is nevertheless good enough to trace extinctions of the order of $\mathrm{A}_{V} \sim 20 \mathrm{mag}$, which is the maximum value found along the north-eastern filamentary structure and northern regions.

The average extinction of the diffuse low density remnant cloud covering most of the $\mathrm{W} 40 \mathrm{H}$ II region is found to be $A_{V} \sim 15 \mathrm{mag}$. Note that the low extinction cavity around the IRS sources in Figure 3 displays $\mathrm{A}_{V} \leq 12 \mathrm{mag}$ which agree well with the previous extinction estimates for the dominant IRS sources $\left(A_{V} \sim 8-10 \mathrm{mag}\right)$ in the region (Smith et al. 1985; Shuping et al. 1999; Rodríguez et al. 2010).

\subsection{Surface density}

We carried out the YSO surface density analysis of the region using the nearest neighbour (NN) method (Casertano \& Hut 1985; Schmeja et al. 2008; Schmeja 2011). Figure 4 shows contours for 50 and 20 NN density overlaid on PACS $160 \mu \mathrm{m}$ image and IRAC $3.6 \mu \mathrm{m}$ image, respectively. A large value of $\mathrm{NN}$ is used to examine large scale structures, while a lower value of $\mathrm{NN}$ is more sensitive to smaller-scale density variations and is used to trace sub-structures. This can be thought of some kind of smoothing process. Here, the 50 NN density contours are fairly circularly symmetric, and were thus used to calculate the cluster radius (as opposed to using $20 \mathrm{NN}$ map). The dense central region for $50 \mathrm{NN}$ map was fitted by a Gaussian profile whose FWHM comes out to be $\sim 3^{\prime}$ $(\sim 0.44 \mathrm{pc}$ at a distance of $500 \mathrm{pc})$. There are 170 YSOs (from Section 3.1) within this cluster radius. The 20 NN density contours (being more sensitive to local density fluctuations) reveal the sub-structures of the cluster. The contours mirror the filamentary structures to a considerable degree showing that the filaments also contain YSOs, albeit at low densities. The centers of both 50 and 20 NN density contour maps are coincident with IRS 1A. Figure 5 shows the cumulative frequency distribution and the histogram for the $20 \mathrm{NN}$ and $50 \mathrm{NN}$ distances in solid black line/curve and dark-grey dotted line/curve, respectively. As is expected, each feature (kink, peak, dip, etc) which is observed in $50 \mathrm{NN}$ histogram/frequency polygon at, say, " $\mathrm{x}$ " NN distance, is observed at $\sim$ " $\mathrm{x} / 2$ " $\mathrm{NN}$ distance in $20 \mathrm{NN}$ histogram/frequency polygon. The cluster radius has been marked in vertical dashed line. The cluster radius seems to be near an inflection point in the rising portion of the $50 \mathrm{NN}$ frequency polygon.

\subsubsection{Comparison with other regions}

Schmeja et al. (2008) have carried out an analysis of clusters in nearby star-forming regions - Perseus, Serpens, and Ophiucus molecular clouds - using Spitzer 

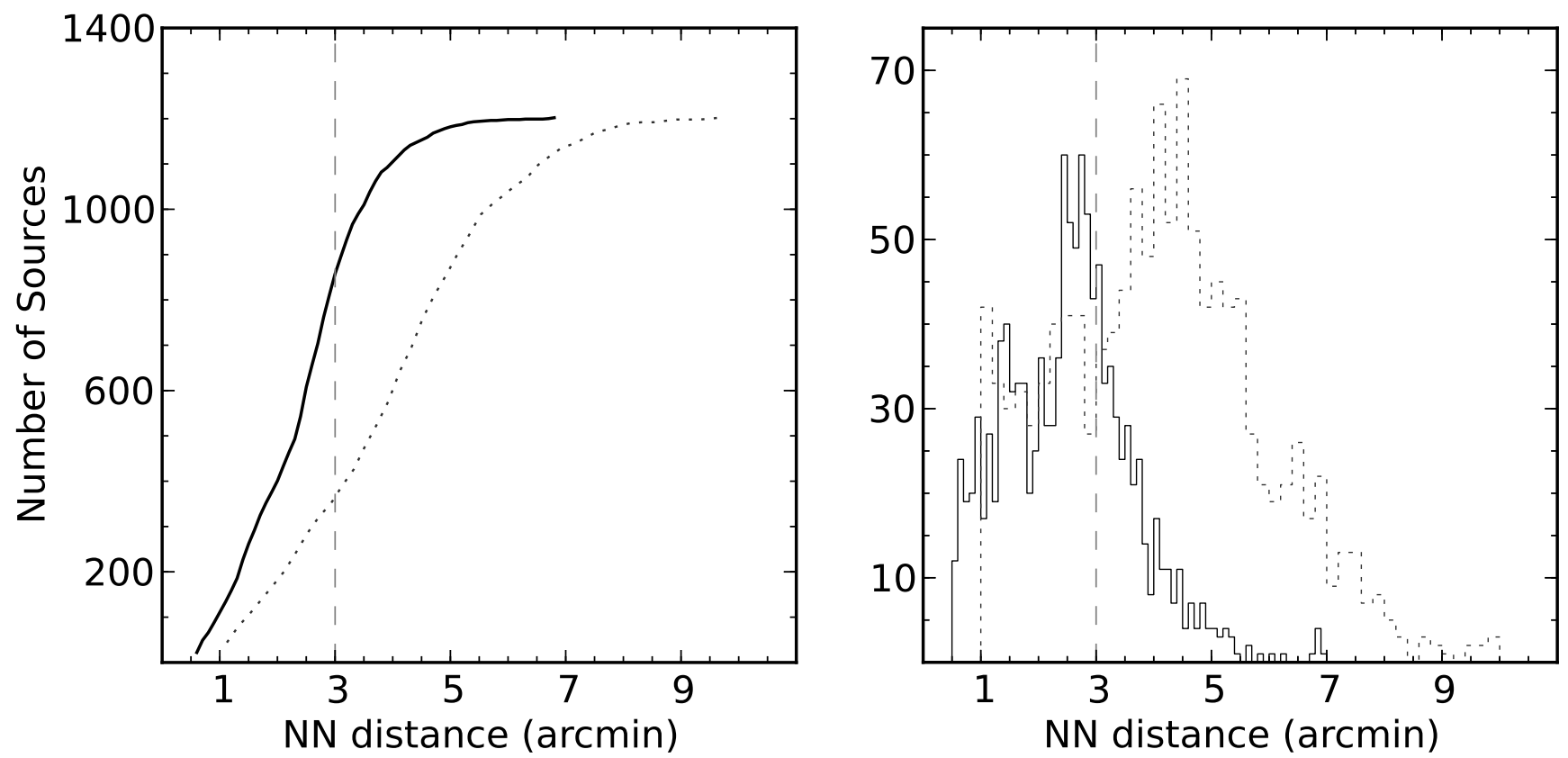

FIG. 5.- (left) The frequency polygon for the cumulative frequency distribution of the nearest neighbour (NN) distances, and (right) the histogram of the NN distances. The NN distances are in arcminutes. The solid black line/curve is for 20 NN distances, while the dark-grey dotted line/curve is for $50 \mathrm{NN}$ distances. The binsizes taken are 0.10 and 0.20 for $20 \mathrm{NN}$ and $50 \mathrm{NN}$, respectively. The vertical grey dashed line marks the cluster radius as calculated from $50 \mathrm{NN}$ map in Figure 4.

data. The cluster peak for the W40 region $(\sim 650$ $\mathrm{pc}^{-2}$ for $20 \mathrm{NN}$ ) seems to be much higher than the clusters in these molecular clouds, except for that in Serpens Core(A) (which is $1045 \mathrm{pc}^{-2}$ ), and comparable to L1688 in Ophiucus (which is $509 \mathrm{pc}^{-2}$ ); while the cluster radius is much smaller (at least a factor of $\sim 0.5$ or more lesser) than all of them, except for that of Ophiucus Centre cluster (which has a radius of $0.52 \mathrm{pc})$. From the compilation of Lada \& Lada (2003) for different clusters (though it must be kept in mind that this is pre-Spitzer and mostly just using NIR), we can see that among the few clusters at similar distances and with similar cluster radii (e.g. L1641N, L1641C, MWC 297, S 106), the star count is mostly much lower than for W40 region (which is 170). Even among regions at larger distances and similar radii (e.g. $01546+6319, \quad 02407+6047, \quad 02497+6217, \quad 02541+6208$, IRAS 06068+2030, IRAS 06155+2319, MWC 137), the star counts are much lower.

\subsection{Spatial distribution and various features}

The YSOs are not in particular found to be correlated with the lobes of the bipolar nebula, suggesting that the bipolar nebula is relatively young, and not enough material has accumulated along the edge of the lobes to lead to star formation. There appears to be a prominent compact nebula (marked with a black arrow on $3.6 \mu \mathrm{m}$ image in Figure 4) located at the edge of the southern lobe. The source associated with this compact nebula was not classified as any YSO (though there are Class II/III YSOs in its vicinity). It is likely to be a reflection nebula illuminated by the embedded star, and seems to have been overrun by the expanding southern wall of the bipolar nebula.

Figure 6 shows the zoomed-in view of the central re- gion with overlaid YSOs. Class I and Class II/III sources have been marked in blue squares and green circles, respectively. While Class II/III sources are distributed throughout the image, the Class I sources are located mostly along the filamentary structures extending upto beyond the cluster radius (details in Section 4.1). Additionally, protostars and starless cores from Maury et al. (2011) have been marked in blue diamond symbols and crosses, respectively. We would like to note here that none of the Class 0/I sources or starless cores from Maury et al. (2011) had any YSO counterpart from our analysis within $1^{\prime \prime}$ matching radius, probably because these Maury et al. (2011) sources are younger (and/or are oriented edge-on) as opposed to the YSOs identified in Section 3.1 which are Class I or Class II/III sources. It is possible that smoothing by Maury et al. (2011) to $13^{\prime \prime}$ beam - while we are only matching upto $1^{\prime \prime}$ here - might be the cause of mismatch. Moreover, the classification by Maury et al. (2011) is using envelope mass vs. bolometric luminosity diagram, inducing further uncertainty.

The high-mass IRS sources have been marked with arrows and labelled. IRS $1 \mathrm{~A}$ South - the main ionizing source - was found, using CC/CM diagrams, to exhibit an IR excess emission. This could be because it is located in the central region surrounded by high density material. IRS $2 \mathrm{~B}, 3 \mathrm{~A}$, and 5 (other high-mass sources, but of comparatively much lower mass than $1 \mathrm{~A}$ South) were found to have a negative SED slope, with the respective spectral indices being $\sim-1$ for IRS $2 \mathrm{~B},-0.4$ for IRS $3 \mathrm{~A}$, and -1.9 for IRS 5 .

\subsubsection{IRS 5 nebular region}

Another noticeable feature is the arc-shaped nebula around the IRS 5 high mass source. Shuping et al. (2012) have estimated the spectral type of this source as B1 


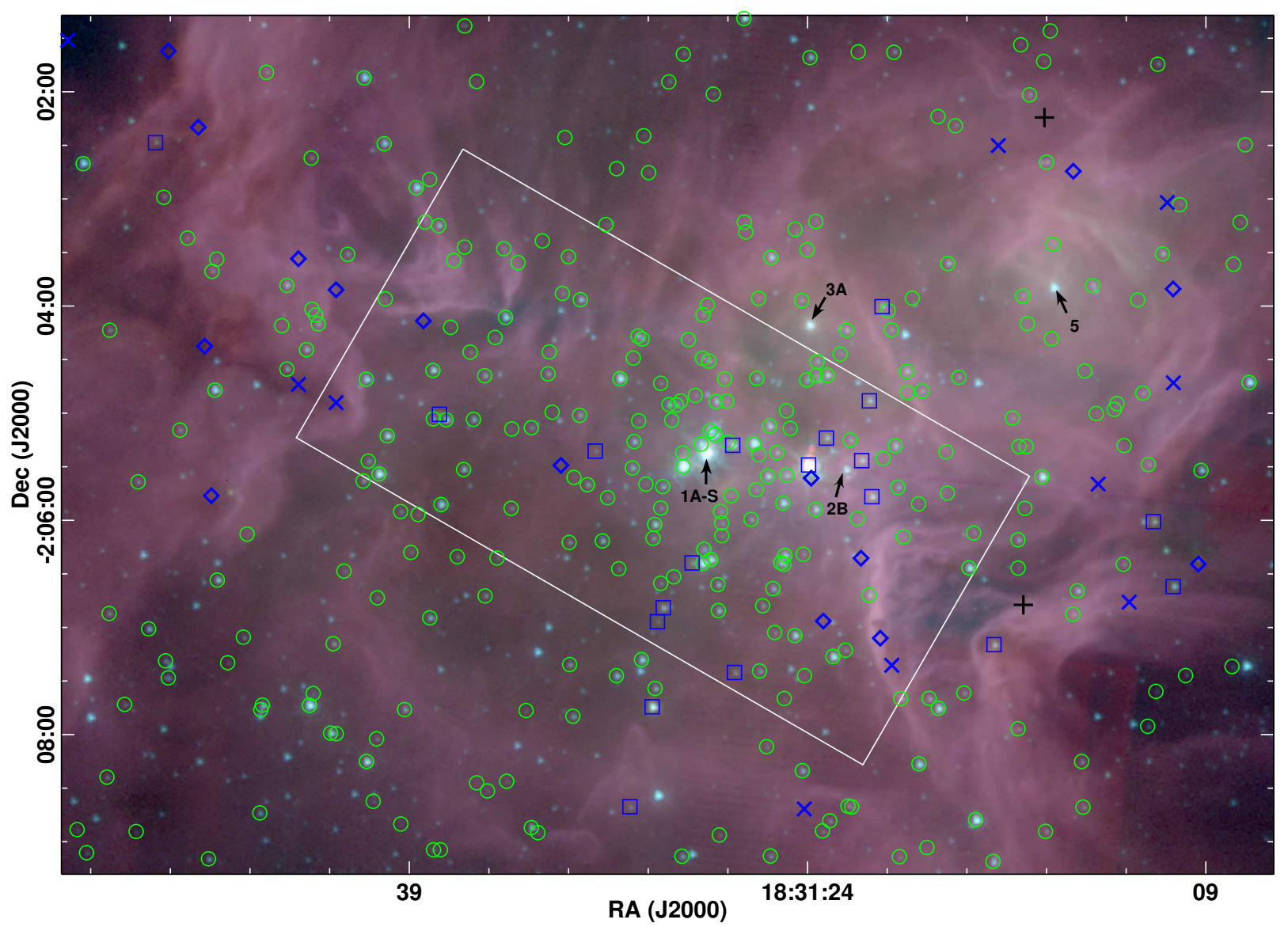

FIG. 6.- Colour composite image of the zoomed-in central region using IRAC $8.0 \mu \mathrm{m}$ (red), $4.5 \mu \mathrm{m}$ (green), and $3.6 \mu \mathrm{m}$ (blue) images. The high-mass IRS sources (1A-South, 2B, 3A, and 5) have been marked by black arrows, while the ${ }^{12} \mathrm{CO}(\mathrm{J}=1-0)$ peaks (Zeilik \& Lada 1978; Smith et al. 1985) have been marked by black plus symbols. Green circles and blue squares denote the Class II/III and Class I sources, respectively. Blue diamond and blue cross symbols denote protostars and starless cores, respectively, from Maury et al. (2011). The white rectangle denotes possible "hub" region (see Section 4.1).

type. The protostars and starless cores from Maury et al. (2011) seem to be distributed along the circumference of this arc-shaped nebula. This probably indicates that material has been pushed by the expansion of $\mathrm{H}$ II region of IRS 5 and collection of dense material due to it has led to the formation of these protostellar sources and cores.

\subsubsection{Bright-Rimmed Clouds}

The zoomed-in central region shown in Figure 6 with overlaid sources shows the presence of manifold brightrimmed cloud (BRC) like structures with all their 'heads' pointing in almost the same direction - towards the center - suggestive of the fact that they could have been carved out by the radiation of central high-mass star(s); though alternatively, these illuminated bright-rims might just be the borders of normal density filaments carved out by the ionizing radiation and hence pointing towards the central region. There are very few YSOs associated with the heads of these BRCs, unlike what has been observed in many regions (Ogura 2010; Chauhan et al. 2009).

\subsubsection{Comparison with $H \alpha$ emission}

Figure 7 shows a colour composite image made using PACS $160 \mu \mathrm{m}$ (red), IRAC $3.6 \mu \mathrm{m}$ (green), and $\mathrm{H} \alpha$ image (blue) from SuperCOSMOS $\mathrm{H} \alpha$ survey (Parker et al. 2005). The dashed white box in the image marks the IRS 5 arc-shaped nebular region. The image shows the current star formation activity (traced by $\mathrm{H} \alpha$ emission) vis-a-vis the dispersed molecular material by the high-mass sources (traced by PAH in $3.6 \mu \mathrm{m}$ band). As can clearly be seen, there is no correlation between $\mathrm{H} \alpha$ emission and the $3.6 \mu \mathrm{m}$ emission; though this could partially be the effect of variable extinction. The $\mathrm{H} \alpha$ emission regions are also not correlated with the location of our detected YSOs. $\mathrm{H} \alpha$ is mainly present in the IRS 5 nebular region, and in selected portions to the south of the midriff. Some of the $\mathrm{H} \alpha$ emission is correlated with BRCs and the so-called elephant trunks (to the east). It is likely that the stellar objects in these $\mathrm{H} \alpha$ emission regions are in extremely young stages.

\subsection{Estimating masses}

We determined the mass of the cloud within the central cluster radius and the IRS 5 region using the extinction map, assuming the empirically determined gas-todust ratio of $\left\langle N\left(H_{2}\right) / A_{V}\right\rangle=0.94 \times 10^{21}$ molecules $\mathrm{cm}^{-2}$ mag $^{-1}$ (Ciardi et al. 1998; Lilley 1955; Jenkins \& Savage 1974; Bohlin et al. 1978). This expression has been derived assuming that the total-to-selective extinction ratio $\left(\mathrm{R}_{V}\right)$ is 3.1, and that most of the gas is in form of molecular hydrogen (Frerking et al. 1982; Krumholz et 


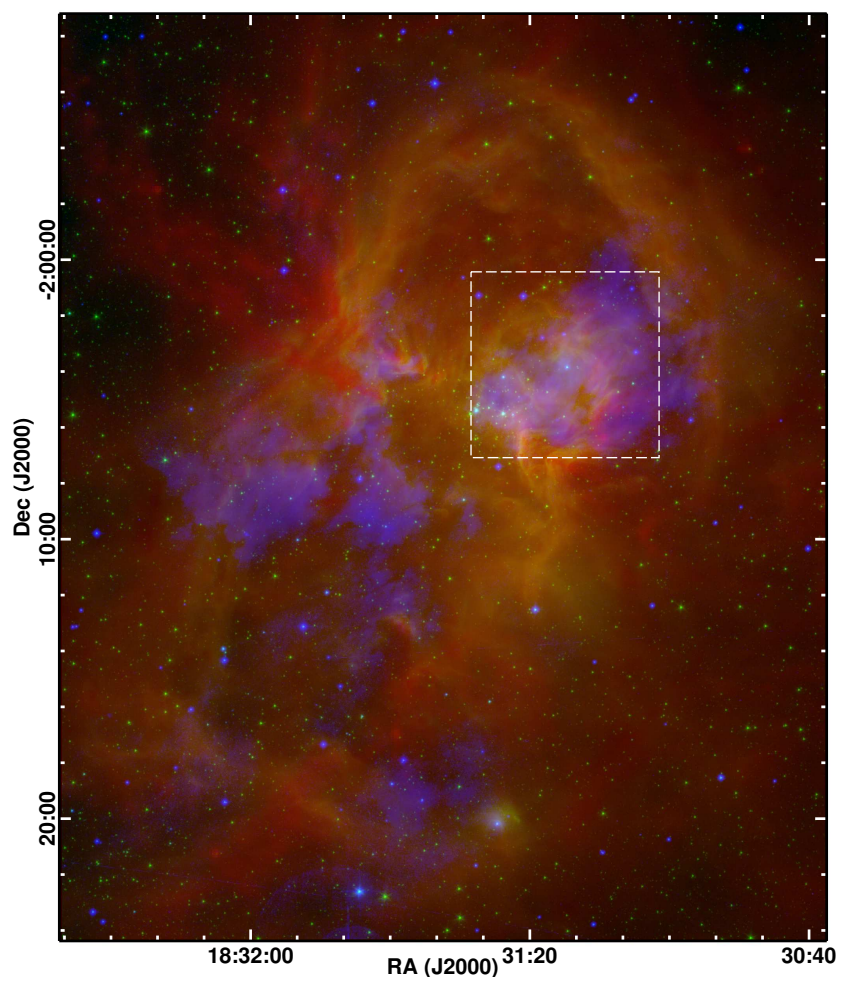

FIG. 7.- Colour composite image made using PACS $160 \mu \mathrm{m}$ (red), IRAC $3.6 \mu \mathrm{m}$ (green), and $\mathrm{H} \alpha$ image (blue). Dashed white box shows the IRS 5 nebular region.

al. 2011). For the central cluster region (which has a radius of $3^{\prime}$ centered on IRS 1A), first the column density at each pixel (of size $20^{\prime \prime}$ ) was determined using the $\mathrm{A}_{V}$ value in that pixel. Thereafter, column density values in all the pixels within the cluster area were integrated to obtain the total mass in that area. Same method was used to calculate the mass of the IRS 5 region - with the area taken as that extending from the edge of the central cluster circumference till approximately the edge of the arc-shaped nebula. The areas used for the calculation of masses in the central cluster and the IRS 5 region are mutually exclusive. The resulting masses obtained for the central cluster region and IRS 5 region were $\sim$ $126 \mathrm{M}_{\odot}$ and $\sim 71 \mathrm{M}_{\odot}$, respectively. These estimates are most likely lower limits as a not unsignificant fraction of the molecular gas might have been expelled during the course of evolution of the cluster. Also, some of the gas will be present in form of the ionised gas. Another caveat to be kept in mind is that $\mathrm{R}_{V}$ can, in general, have wide variations (Mathis 1990).

\section{MORPHOLOGY OF THE REGION}

\subsection{Filamentary Structures}

Figure 8 shows Herschel PACS $160 \mu \mathrm{m}$ image of the larger region $\left(\sim 45.5^{\prime} \times 63.0^{\prime}\right)$ with overlaid YSOs, and radio sources (in green plus symbols) from Pirogov et al. (2012). The location of the molecular clump TGU 279P7 from Dobashi et al. (2005) has been marked in a green cross. Apart from the circular lobes which join at the midriff, filamentary structures are also seen emanating towards north-eastern and southern directions from the central region. In Figure 8, three prominent parsec-scale filaments, marked on the image, can clearly

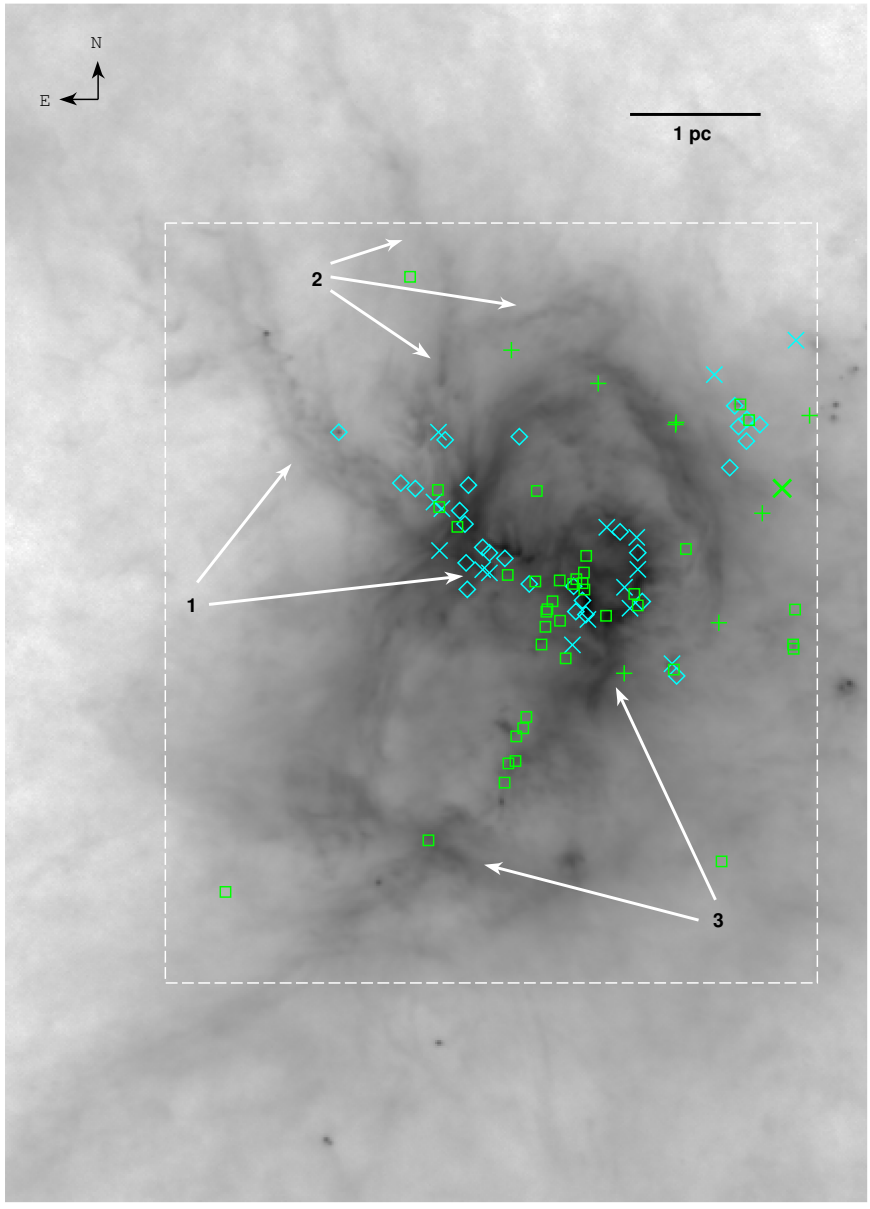

FIG. 8.- Herschel PACS $160 \mu \mathrm{m}$ image overlaid with the youngest sources in the region. Cyan diamond and cyan cross symbols denote protostars and starless cores, respectively, from Maury et al. (2011). Green square symbols denote Class I sources. Prominent filaments have been marked with arrows and labelled. Green plus symbols mark the radio sources from Pirogov et al. (2012). Green cross marks the location of the molecular clump TGU 279P7 from Dobashi et al. (2005). White dashed box marks the area of our analysis in the paper.

be made out. Due to high extinction in the region and by virtue of their intrinsically dense nature, the filamentary structures start becoming visible in emission only at Herschel PACS 100 and $160 \mu \mathrm{m}$ bands and at submillimeter SPIRE wavelengths (see Könyves et al. 2010, for the image of the larger region encompassing W40). They can also be seen in absorption against the dominant $\mathrm{PAH}$ (plus the continuum) emission at $11.3 \mu \mathrm{m}$ and $12.7 \mu \mathrm{m}$ in WISE $12 \mu \mathrm{m}$ band image (not shown here).

Filament 1 is about $\gtrsim 2.4 \mathrm{pc}$ long, and seems to be splitting into two parts near the midriff where it ploughs into the cloud. Possible signatures are the two bow shocks (the southern portion of filament 1 , marked with an arrow) visible here (also see Figure 4(left) for clarity). Filament 2, which is comparatively diffuse, extends upto $\gtrsim 1.2 \mathrm{pc}$ to the north of the central region, where it bifurcates into a filament to the north $(\gtrsim 1.6 \mathrm{pc}$ in length) and the other to the west ( $\gtrsim 1$ pc in length). Filament 3 is the longest visible structure, extending for $\gtrsim 3 \mathrm{pc}$ from the western part of the central region, and seemingly cutting across the southern lobe of the bipolar nebula. According to the column density map of Könyves 


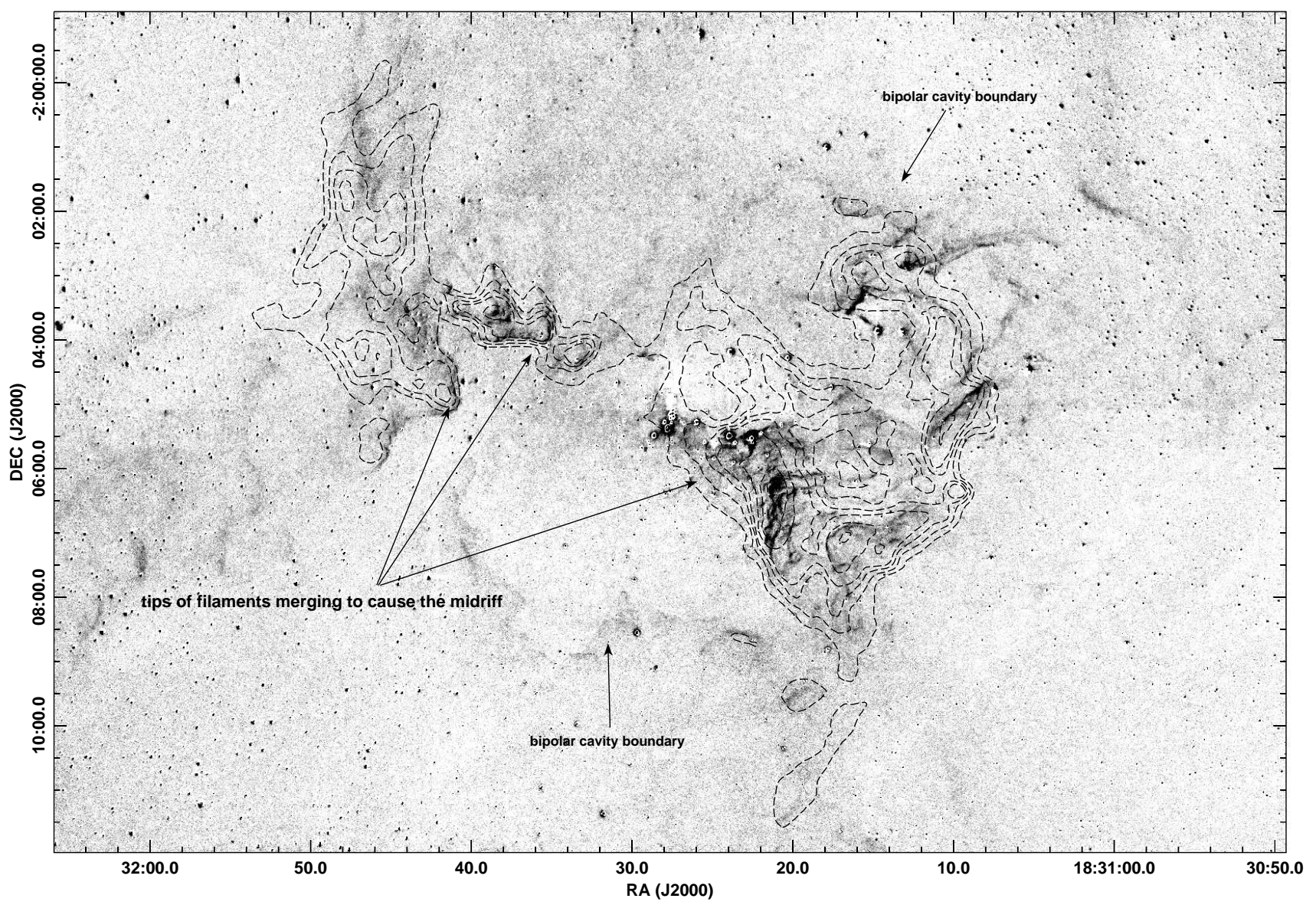

FIG. 9. - Continuum-subtracted $2.12 \mu \mathrm{m} \mathrm{H}_{2}$ narrow-band image of the region in inverted grey-scale, with overlaid PACS $160 \mu \mathrm{m}$ contours. The prominent features have been marked.

et al. (2010), the value throughout - for all the three filamentary structures - is of the order of $N_{H_{2}} \sim 10^{22} \mathrm{~cm}^{-2}$. The filamentary structures are also seen in dust temperature as well as extinction maps (Bontemps et al. 2010, see their Figures 1 and 3).

Figure 8 also shows overlaid YSOs, with Class I sources, protostars, and starless cores marked in green squares, cyan diamond symbols, and cyan crosses, respectively. As can be seen on the image, most of the sources seem to be distributed along two radially outward filaments 1 and 3 from the central region which has a concentration of these YSOs. Filament 2 hardly contains any of these youngest sources, probably because of its diffuse nature, suggesting that it is most likely still not Jeans critical.

Myers (2009) had proposed a hub-filament model according to which a central 'hub' with a column density of $\sim 10^{22} \mathrm{~cm}^{-2}$ radiates filaments which can be seen upto a column density of $\sim 10^{21} \mathrm{~cm}^{-2}$. It was suggested that the hub should have a minimum surface density of 25 YSOs $\mathrm{pc}^{-2}$. The filamentary structures of the W40 region resemble this hub-filament structure. A possible 'hub' region is marked in Figure 6 by a white rectangle. This 'hub' region has a stellar density of $\sim 358$ YSOs $\mathrm{pc}^{-2}$ (total number of YSOs divided by total area), while the mean column density over this hub region is $N_{H_{2}} \sim 1.8$ $\times 10^{22} \mathrm{~cm}^{-2}$ (Maury et al. 2011). Among the sources from our analyses and literature, a total of 12 Class I sources, 6 protostars, and 126 Class II/III sources are located in this 'hub'. This translates to the fraction of youngest sources (Class I/protostars) being $\sim 0.125$, and thus, according to the criteria used by Myers (2009) (that this fraction should be $\geq 0.1$ ), this region can be deemed "young".

There are filaments to the west of this bipolar nebula which are a part of Serpens South cluster (not shown here). Though they appear to be spatially correlated with W40 region, this could be a projection effect; as the distance to Serpens South is about 260 pc (Nakamura et al. 2011), while the distance to W40 - though with varying values based on different techniques - has been estimated to be about 500 pc (Shuping et al. 2012).

\section{2. $\mathrm{H}_{2}$ narrow-band}

In Figure 9 we display the continuum-subtracted $\mathrm{H}_{2}$ narrow-band image using inverted grey scale. The brightest emission from the Herschel PACS $160 \mu \mathrm{m}$ image is overlayed using dashed contours. Several important features are marked on this figure which are explained below. Comparing $\mathrm{H}_{2}$ emission with $160 \mu \mathrm{m}$ contours indicates that most of the observed $\mathrm{H}_{2}$ emission traces the boundaries of filaments and dense gas traced by the $160 \mu \mathrm{m}$ contours. The $\mathrm{H}_{2}$ emission also reveals a faint bipolar shaped cavity centered on the W40 embed- 


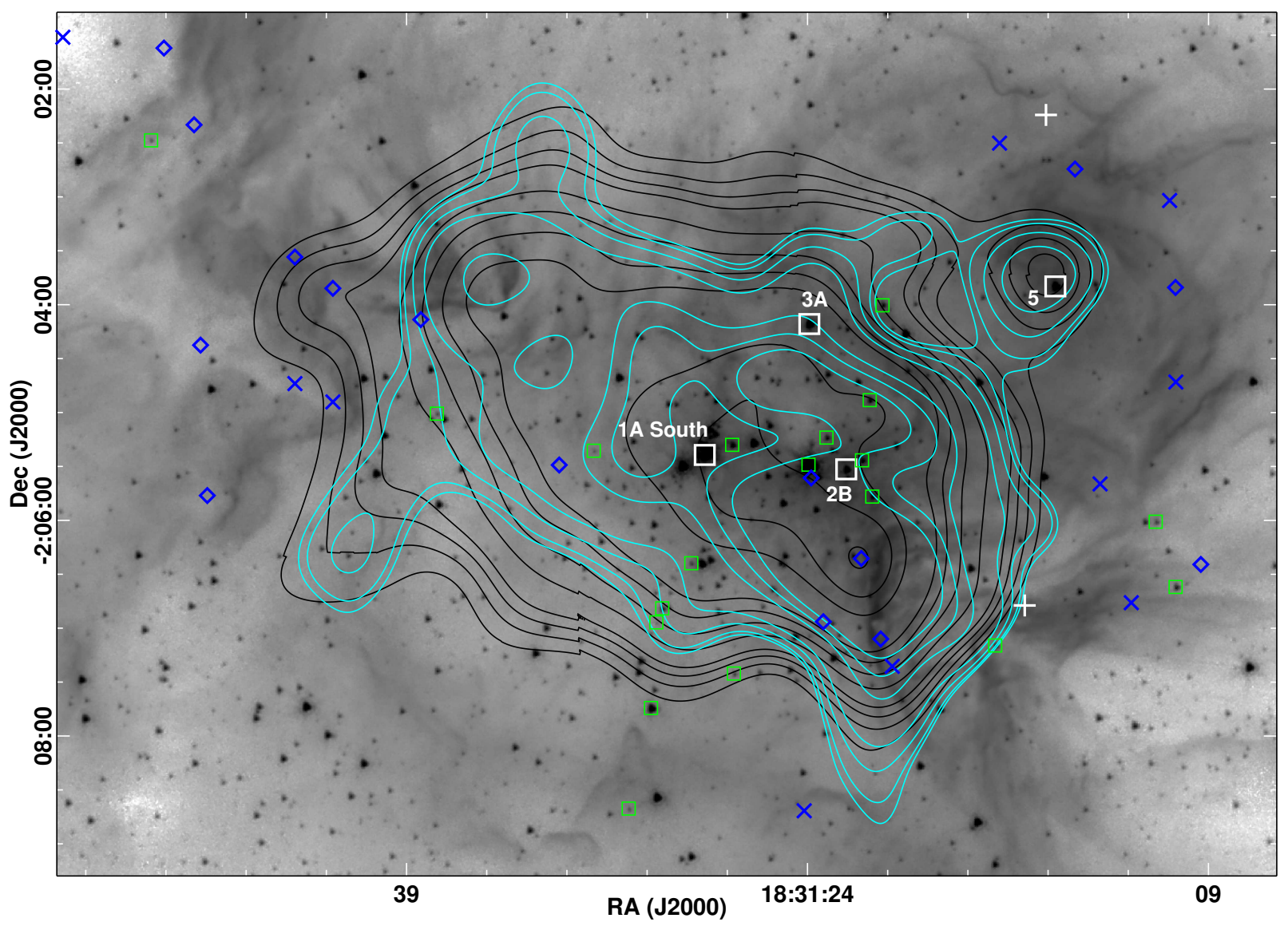

FIG. 10. - Spitzer $3.6 \mu \mathrm{m}$ image overlaid with early type sources, YSOs and radio contours. $1280 \mathrm{MHz}$ and $610 \mathrm{MHz}$ contours have been marked in black and cyan, respectively. White squares with respective labels denote the early type stars in the region from Shuping et al. (2012). Plus symbols mark the ${ }^{12} \mathrm{CO}(\mathrm{J}=1-0)$ peaks (Smith et al. 1985). Blue diamond and blue cross symbols denote protostars and starless cores, respectively, from Maury et al. (2011). Green square symbols denote Class I sources. $1280 \mathrm{MHz}$ contours are drawn at 4,6 , $8,10,15,20,25,30,50,60$, and $70 \sigma(\sim 6.34 \mathrm{mJy} /$ beam $)$, while $610 \mathrm{MHz}$ contours have been drawn at 4, 5, 7, 10, 20, 25, and 30 $\sigma(\sim$ $5.52 \mathrm{mJy} /$ beam).

ded cluster. The northern lobe of this cavity is clearly brighter than the southern lobe, however, the $160 \mu \mathrm{m}$ emission is also stronger in this northern region. BRCs can be seen along the southern boundary of this bipolar shaped cavity in IRAC images (see Figure 6). The morphology of the observed $\mathrm{H}_{2}$ emission features are indicative of fluorescent excited emission in most of the observed field. We note that the arc-shaped bipolar cavity in the northern region, partially surrounding the IRS 5 source, is also the region where several starless cores were mapped by Maury et al. (2011) (see Section 3.4). It may therefore represent a dense shell swept by the radiation from the central embedded cluster. While the lack of collimated bipolar features indicate absence of shocked emission in general, confirmation of the fluorescent or shocked nature for the diffuse $\mathrm{H}_{2}$ emission is not possible without obtaining spectroscopic observations.

\subsection{Radio Morphology}

Figure 10 shows the Spitzer $3.6 \mu \mathrm{m}$ image of the central portion of the W40 region. Overlaid are the $610 \mathrm{MHz}$ and $1280 \mathrm{MHz}$ radio contours from GMRT. The earlytype stars in the region - IRS 1A South, 2B, 3A, and 5 have been marked with white squares and labelled. The ${ }^{12} \mathrm{CO}(\mathrm{J}=1-0)$ peaks from Smith et al. (1985) have been marked with plus symbols. It can clearly be seen that one of the ${ }^{12} \mathrm{CO}(\mathrm{J}=1-0)$ peaks lies at the western edge of the $\mathrm{H}$ II region. The ${ }^{13} \mathrm{CO}(\mathrm{J}=2-1)$ and ${ }^{13} \mathrm{CO}(\mathrm{J}=3$ 2) maps from Zhu et al. (2006) also reveal the presence of molecular cloud to the west of ionizing region. This molecular cloud to the west of the $\mathrm{H}$ II region has also been observed in various molecular lines and in continuum at millimeter wavelengths (Pirogov et al. 2013). The region thus appears to be density bounded to the west as is evidently borne out by the CO isotopologues' peaks. But, there is no such constraint towards the east, leading to the proliferation of ionizing photons towards this direction. This is indicative of blister morphology, also referred to as champagne flow (Tenorio-Tagle 1979; Whitworth 1979), in the region. The radio contours depicted on the image also support this blister morphology for the region, with the head of the ionized flow towards the west (density-bounded), and an extended emission towards the east. The peak of $1280 \mathrm{MHz} \mathrm{H}$ II emission is about $1.24^{\prime}$ to the south-west of the peak at $408 \mathrm{MHz}$ from Goss \& Shaver (1970), but this could be due to their lower beamsize $\left(\sim 3^{\prime}\right)$. As can be seen in Figure 10, there are no Spitzer Class I YSOs near this peak, only Class 0/I sources or starless cores from Maury et al. (2011) are 
seen. Also, one of the Class 0/I sources from Maury et al. (2011) coincides with the $1280 \mathrm{MHz}$ radio peak. This is a probable indication of some high-mass star formation going on in this region, which is also supported by the outflow seen by Zhu et al. (2006). The timescale of the outflow from Zhu et al. (2006) $\left(\sim 6 \times 10^{4} \mathrm{yr}\right)$ is of the order of the age estimate from Maury et al. (2011) ( $\left.4-9 \times 10^{4} \mathrm{yr}\right)$ of Class 0/I sources. However, it is plausible that the "outflow" observed by Zhu et al. (2006) might simply be the material swept-up and pushed into the bipolar shaped nebula. The $\mathrm{H}$ II emission does not extend all the way up to the edge of the bipolar lobes (which marks the edge of the photo-dissociation region) or trace them at GMRT sensitivity levels. The extent of the central $\mathrm{H}$ II region is almost same as the cluster radius at half-peak surface density (see Section 3.4 and Figure 4), which is expected. The central ionizing region is limited to $\sim 2^{\prime}(\sim 0.29 \mathrm{pc})$ on either side of the midriff of the bipolar lobes. Though isolated, non-thermal radio emission sources have been discovered by Pirogov et al. (2012) (see Figure 8), it seems unlikely that they are related to the W40 region due to their non-thermal nature. Another feature of note is the sub-region around IRS 5 which shows distinct radio emission with a roughly circularly symmetric ionisation region around this source.

\subsection{Overall Structure}

The presence of these various morphological structures support the schematic for this region by Vallee (1987, see their Figure 2). The high-mass stars formed are probably located at the edge of the parental giant molecular cloud towards the observer, and as these sources have ionized the natal medium, the whole $\mathrm{H}$ II region has broken out of the parental cloud. The filaments seem to be emanating from the midriff of the W40 region. This suggests that the density was high along the equatorial axis, as compared to the polar directions, leading to a high density contrast. Thus the Lyman continuum radiation has escaped along the polar directions, flinging the ionised gas (which forms the bipolar nebula) partially towards the observer. This flung-off material seems to have encountered more resistance towards the north - as seems likely by the smaller diameter of the northern lobe, more extincted regions towards the north, and brighter $160 \mu \mathrm{m}$ emission from the northern lobe (see Figures 8). This probably indicates that the main body of the cloud lies to the north of the W40 midriff region. The initial orientation and location of the high-mass stars seem such that this presence of molecular cloud to the north has proven harder to disrupt, while the southern thin shell of molecular material (between the high-mass stars and the ISM) has been blown away relatively easily. We note that the southern lobe could be appearing larger than the northern lobe due to the projection effect from the viewing angle, i.e. the region might not be being viewed exactly face-on, but from a non-zero inclination angle. As has also been evidenced in Beaumont \& Williams (2010) for various other regions, these northern and southern lobes could be more like rings than spheres, opening up the possibility that the molecular cloud could be oblate or sheet-like. The morphology of the filamentary structure shows its complete extent till the filaments join the central region. Since the filaments can be seen right till they join the midriff, they are most likely in front of the progenitor molecular cloud.

\section{PHYSICAL PARAMETERS}

\subsection{Overall Region}

Radio continuum observation data was used to derive the characteristic physical parameters like emission measure, electron density, dynamical age, and strömgren radius for the region. Since this region contains multiple radio sources (Rodney \& Reipurth 2008), we carried out a global analysis using the low resolution images $\left(\sim 45^{\prime \prime} \times\right.$ $\left.45^{\prime \prime}\right)$ to get an estimate of the parameters. Integrated flux density was calculated for both the frequencies within $4 \sigma$ contours using the AIPS task "TVSTAT"; giving us a value of $4.07 \mathrm{Jy}$ and $7.55 \mathrm{Jy}$ at $610 \mathrm{MHz}$ and $1280 \mathrm{MHz}$, respectively. Thereafter, using the model for free-free emission of Mezger \& Henderson (1967), the flux density in a region is given by (adapted from Mezger et al. 1967):

$$
\begin{gathered}
S_{\nu}=3.07 \times 10^{-2} T_{e} \nu^{2} \Omega\left(1-e^{-\tau(\nu)}\right) \\
\tau(\nu)=1.643 \times 10^{5} a T_{e}^{-1.35} \nu^{-2.1} n_{e}^{2} l
\end{gathered}
$$

where, $S_{\nu}$ is the integrated flux density in $\mathrm{Jy}, T_{e}$ is the electron temperature of the ionized core in $\mathrm{K}, \nu$ is the frequency in $\mathrm{MHz}, n_{e}$ is the electron density in $\mathrm{cm}^{-3}, l$ is the extent of the ionized region in pc, $\tau$ is the optical depth, $a$ is the correction factor, and $\Omega$ is the solid angle subtended by the beam in steradian here. $n_{e}^{2} l$, called the emission measure, measures the optical depth in the medium (in $\mathrm{cm}^{-6} \mathrm{pc}$ ). For our calculation, we took $T_{e}$ to be 8500 K (Shaver \& Goss 1970; Quireza et al. 2006), and thus the correction factor $a$ as 0.99 (Mezger \& Henderson 1967, Table 6). Using the two data points for 610 $\mathrm{MHz}$ and $1280 \mathrm{MHz}$, we fit the above equations using a non-linear regression with emission measure $\left(n_{e}^{2} l\right)$ as a free parameter (Figure 11(a)). This yielded the value of emission measure as $1.63 \pm 0.09 \times 10^{6} \mathrm{~cm}^{-6} \mathrm{pc}$. The extent $(l)$ of the central $\mathrm{H}$ II region is approximately $7.0^{\prime}$, equivalent to about $1.02( \pm 0.41)$ pc at a distance of 500 $( \pm 200)$ pc; and thus the electron density turns out to be $\sim 1265 \pm 218 \mathrm{~cm}^{-3}$ for this central region.

The Lyman continuum luminosity (photons $\mathrm{s}^{-1}$ ) required for ionizing the gas was calculated by using the formulation of Moran (1983, see their Equation 5) for the optically thin regime. As is evident from Figure 11(a), $610 \mathrm{MHz}$ and $1280 \mathrm{MHz}$ emission lie in the optically thick and thin regimes, respectively. Therefore, using the flux density of 7.55 Jy estimated at $1280 \mathrm{MHz}$, the Lyman continuum luminosity was calculated to be $\sim 1.67 \times 10^{47}$ photons $\mathrm{s}^{-1}$. Shuping et al. (2012) have estimated that the radio emission is dominated by an O9.5 type star (IRS 1A South, see Figure 10) with a luminosity of 6.3 $\times 10^{47}$ photons $\mathrm{s}^{-1}$. They place an upper limit on the Lyman continuum luminosity to be $1.6 \times 10^{48}$ photons $\mathrm{s}^{-1}$. Smith et al. (1985), using IR and low resolution $(\sim$ $\left.11^{\prime}\right)$ radio observation of Altenhoff et al. (1970) inferred a luminosity of $\sim 1.5 \times 10^{48}$ photons $\mathrm{s}^{-1}$. Our Lyman continuum luminosity estimate differs by a factor of the order of unity from the calculated flux of an O9.5 type star in Shuping et al. (2012) and the tabulated value from Martins et al. (2005). However, it is about $10 \%$ of the value from Smith et al. (1985) and of the upper limit in 

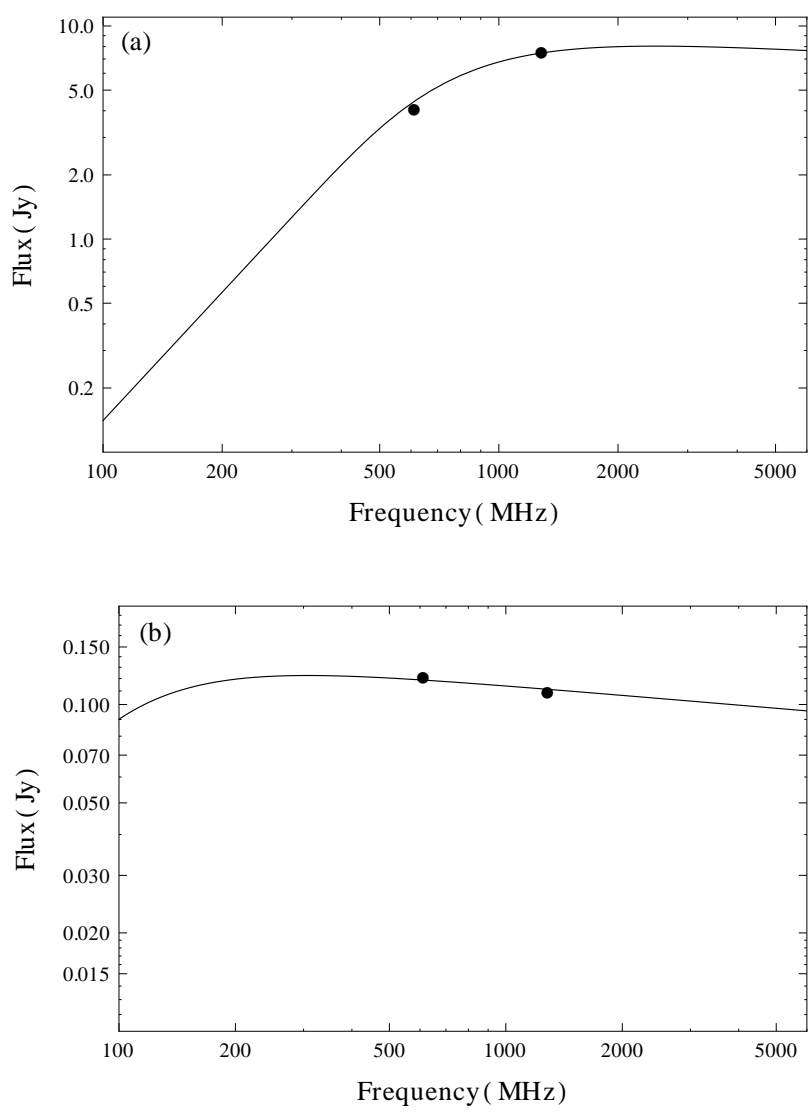

FIG. 11.- The fitted flux density model (solid line) for (a) the entire region, and (b) the IRS 5 region. The data points at 610 $\mathrm{MHz}$ and $1280 \mathrm{MHz}$ have been marked with black solid circles.

Shuping et al. (2012). This could be because of absorption of photons by the dust in the region, implying the presence of dense gas in the central region. As has also been noticed by Kurtz et al. (1999), presence of dust absorption can lead to underestimation of radio luminosity. Arthur et al. (2004) have estimated that dust absorption can range from $60 \%$ upto $96 \%$ of the ionizing photons. Similar underestimation of ionizing flux by radio data has been observed for other regions in Brand et al. (2011); Alvarez et al. (2004). The luminosity calculated here, therefore, is most likely just the lower limit.

In the first stage during the ionization of a region by massive star(s), the ionization front propagates outwards leading to an expansion of the $\mathrm{H}$ II region, till an equilibrium is reached between the number of ionizations and recombinations. The radius of the $\mathrm{H}$ II region at this point, assuming that the ambient medium has spatially uniform density, is given by (Strömgren 1939) :

$$
R_{s}=\left(\frac{3 S_{L y m a n}}{4 \pi n_{o}^{2} \beta_{2}}\right)^{1 / 3}
$$

where $R_{s}$ is called the Strömgren radius (in $\mathrm{cm}$ ). $n_{o}$ is the initial ambient density (in $\mathrm{cm}^{-3}$ ), and $\beta_{2}$ is the total recombination coefficient to the first excited state of hydrogen. The value of $\beta_{2}$, for a $T_{e}$ of $8500 \mathrm{~K}$, was taken to be $2.94 \times 10^{-13} \mathrm{~cm}^{3} \mathrm{~s}^{-1}$ (Ward-Thompson \& Whitworth 2011; Stahler \& Palla 2005). In the second stage of expansion, after the Strömgren radius has been reached, the shock front overtakes the ionization front due to the
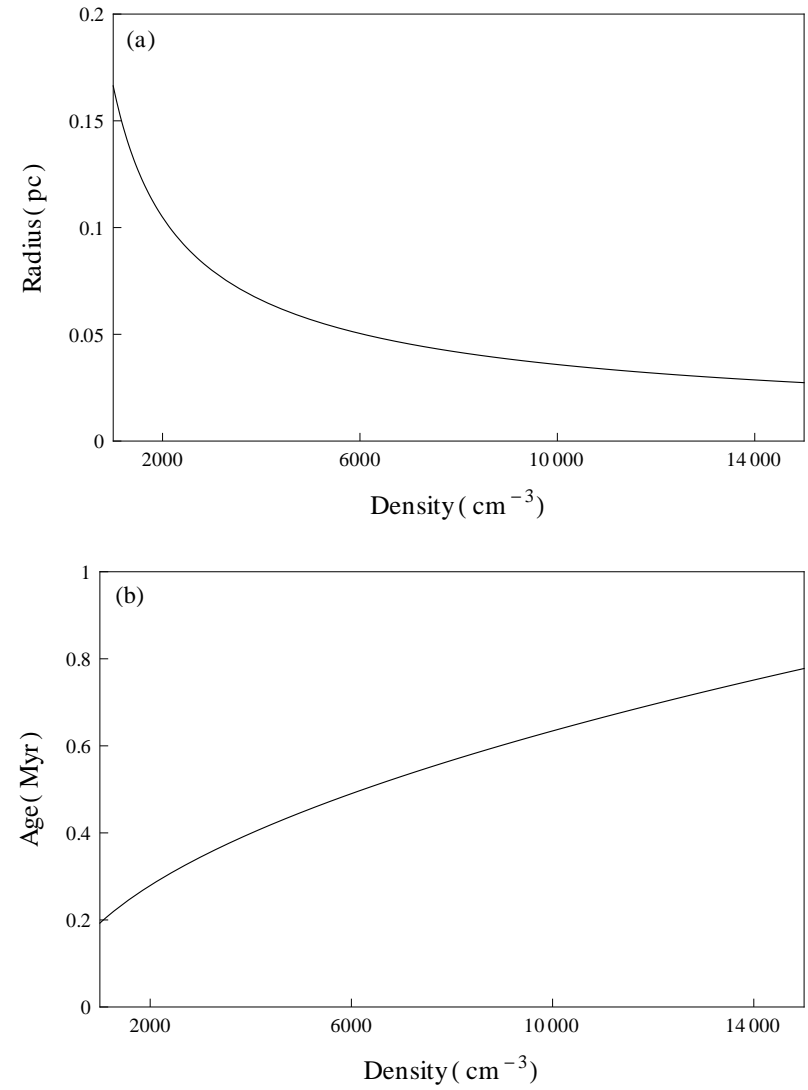

FIG. 12.- Graph showing the variation of (a) Strömgren radius and (b) dynamical age with ambient density for the overall photoionized region.

vast pressure difference between material inside and outside of the ionization front. In this stage, the radius of the $\mathrm{H}$ II region is given by (Spitzer 1978) :

$$
R(t)=R_{s}\left(1+\frac{7 c_{I I} t}{4 R_{s}}\right)^{4 / 7}
$$

where $R(t)$ denotes the extent of the $\mathrm{H}$ II region at time $t$, and $c_{I I}$ is the speed of sound, taken as $11 \times 10^{5}$ $\mathrm{cm} \mathrm{s}^{-1}$ (Stahler \& Palla 2005). Since we have no way of gauging the initial ambient density $\left(n_{o}\right)$ of the molecular cloud, we have plotted the parameters for ambient density ranging from 1000 to $15000 \mathrm{~cm}^{-3}$ to get an estimate of the ranges. Figure 12 shows Strömgren radius $\left(R_{s}\right)$ and dynamical age $(t)$ plotted as a function of initial ambient density of the medium. Strömgren radius varies from $\sim 0.17$ to $0.03 \mathrm{pc}$, while the dynamical age varies from $\sim 0.19$ to $0.78 \mathrm{Myr}$. If we were to take the higher estimates of $S_{\text {Lyman }}$ (the values discussed above), then, for a fixed density, the $R_{s}$ will increase and the $t$ will decrease. It should be noted that there is more than one massive star in the region, and each massive star will have its own Strömgren radius and age. The multiple $3.6 \mathrm{~cm}$ VLA sources found in the region (Rodríguez et al. 2010) will also contribute to the total flux. Also, the radio morphology is not spherical here. Therefore the above calculations should be taken as a representative value for the Strömgren radius and age estimate, and $n_{e}$ should be taken as the averaged out electron density for the ionized region. 


\subsection{IRS 5 Region}

Since radio emission around IRS 5 source is distinct from the rest of the morphology (see Figure 10), we can carry out a separate calculation to determine the physical parameters around this source. For this, we first determined the $610 \mathrm{MHz}$ and $1280 \mathrm{MHz}$ integrated flux density in this region in a circle of $50^{\prime \prime}$ radius centered at the peaks of respective frequencies. This yielded a flux density of 121 and $109 \mathrm{mJy}$ for $610 \mathrm{MHz}$ and $1280 \mathrm{MHz}$, respectively. Rodríguez et al. (2010) have detected IRS 5 source using VLA observations at $3.6 \mathrm{~cm}$. However, they calculated a flux of $0.92 \mathrm{mJy}$ for sub-arcsecond area, while here we are concerned with the extended emission. Another VLA source from Rodríguez et al. (2010, Source 2 in their Table 2) lies close to the IRS 5 source. However, it has a flux of $0.90 \mathrm{mJy}$ (at $3.6 \mathrm{~cm}$ ), and is hence insignificant for our calculation here. Following a similar set of steps as above (Section 5.1), we determined the emission measure by fitting the free-free emission SED (see Figure 11(b)) to be $\sim 2.01 \pm 0.04 \times 10^{4} \mathrm{~cm}^{-6}$ pc. The extent of radio contours for this region is $\sim 100^{\prime \prime}(\sim$ $0.24 \pm 0.10 \mathrm{pc}$ at a distance of $500 \pm 200 \mathrm{pc})$ which gives the electron density as $\sim 288 \pm 55 \mathrm{~cm}^{-3}$.

We estimated the Lyman continuum luminosity using the formulation of Moran (1983) and $1280 \mathrm{MHz}$ data point as in Section 5.1 (in this case, since $610 \mathrm{MHz}$ data point is in the optically thin regime too, it yields similar value), which results in a value of $\sim 2.40 \times 10^{45}$ photons $\mathrm{s}^{-1}$. Comparing $\log$ (Lyman continuum luminosity) (45.38) with the tabulated values from Panagia (1973, Table II), we get the most probable spectral type of IRS 5 as B1V, in agreement with the values from Shuping et al. (2012). The slightly lower value of the calculated Lyman continuum luminosity, as compared to the value from Panagia (1973) is probably due to the absorption of photons by dust in the region.

Though the radio contours are seen within $\sim 50^{\prime \prime}$ radius of the respective radio peaks (Figure 10), it can be seen that the ionised region extends beyond that. This weak extended emission region is traced by $\mathrm{PAH}$ emission at $7.7 \mu \mathrm{m}$ and $8.7 \mu \mathrm{m}$ in IRAC Ch4 and at $3.3 \mu \mathrm{m}$ in IRAC Ch1 (which, it must be kept in mind, also contain continuum emission) (also see Figure 6). Most of Class 0/I sources and starless cores from Maury et al. (2011) are approximately along the circumference of a circle of radius $\sim 80^{\prime \prime}$ centered at respective radio peaks. Therefore, taking the extent to be $160^{\prime \prime}$ and the ambient density $\left(n_{o}\right)$ to be a typical value of $\sim 1000 \mathrm{~cm}^{-3}$ (Stahler \& Palla 2005), we use equation 3 in conjunction with equation 4 to obtain the dynamical age of the $\mathrm{H}$ II region around the IRS 5 arc-shaped nebula to be $\sim 0.11$ Myr. We note that though the ionised region appears more extended than what we have assumed in the above calculation, and the ambient density of the medium could be larger, a higher value of both these parameters will only increase the value of the dynamical age. Therefore, the 0.11 Myr should be considered a lower limit for the dynamical age of this sub-region.

\section{STAR FORMATION SCENARIO}

The nature of the YSOs with their respectively distinct spatial distributions suggest a scenario in which (spontaneous) star formation has taken place at differ- ent epochs for YSOs at different evolutionary stages. It is very likely that Class II/III and the central earlytype sources formed in an earlier epoch as the central region became Jeans critical, followed by the formation of younger sources - Class 0/I sources and starless cores from Maury et al. (2011) and Class I YSOs from our analysis - along filaments in a later epoch. The lifetimes of the dominant early type star in this region (O9.5; Shuping et al. 2012) is of the order of $\sim 10 \mathrm{Myr}$ (Stahler \& Palla 2005), while the lifetimes of Class II/III sources is also of the order of a few Myr. The fact that the two lifetimes are comparable and that this region is not very old, makes it likely that the majority of the Class II/III and central high-mass sources formed at the same epoch. Rodney \& Reipurth (2008), based on the velocity of molecular hydrogen, have opined that star formation might have been initiated several Myrs ago due to an external shock. It is probable that this could have played a role too and affected the earlier epoch of star formation one which led to the formation of Class II/III sources and central high-mass stars. This seems to have been followed by star formation leading to younger YSOs. Alternatively, it is possible that the formation of high-mass stars in the center could have driven a compressional wave into the individual filaments, leading the high-density regions in the filaments to become Jeans critical, thus subsequently initiating the formation of Class I sources, protostars, and starless cores.

The stellar distribution seen has also been observed by Schneider et al. (2012), who, using the Rosette molecular cloud as a template, have done a density analysis to conclude that almost all IR clusters lie at the junction of filaments. Simulations by Dale et al. (2012) also show that the star formation during the course of evolution of molecular clouds to be mostly confined to the filaments and their junctions.

Bontemps et al. (2010), assuming the distance to W40 to be $260 \mathrm{pc}$, have estimated the mass of the molecular cloud (G28.74+3.52) associated with the $\mathrm{W} 40 \mathrm{H}$ II region to be about $1.1 \times 10^{4} \mathrm{M}_{\odot}$ using $2 \mathrm{MASS}$ extinction map. The mass estimated using extinction map depends on the distance as a square of it. Shuping et al. (2012) have recently ruled out distances below $340 \mathrm{pc}$ for this region. Also, mass estimation in high optical depth regions can often get underestimated using this method. So, the mass estimated by Bontemps et al. (2010) is most likely a lower limit and the actual mass could be slightly higher, probably between a few $10^{4} \mathrm{M}_{\odot}-10^{5} \mathrm{M}_{\odot}$.

The IRS 5 region (see Figure 6) also seems to be hosting fresh star formation at the edge of the cavity excavated by the high-mass source. The starless cores and Class 0/I sources from Maury et al. (2011) are found to be distributed along the edge of the $\mathrm{H}$ II region of this arcshaped nebula. The arc of (seemingly) collected molecular material, along the edge of this arc-shaped nebula, has also been observed in various molecular lines (Pirogov et al. 2013), as well as at submillimeter wavelengths Maury et al. (2011) and $\mathrm{H}_{2}$ narrow-band image (see Section 4.2). The farthest YSO from the IRS 5 source in Figure 6 is located at a distance of $\sim 1.8^{\prime}(\sim 0.26 \mathrm{pc})$. Therefore, using the isothermal sound speed of $11 \mathrm{~km} \mathrm{~s}^{-1}$ for the ionised region (see Section 5.1), it can be seen that the high-mass star could have lead to the sweeping-up of material which subsequently might have provided impetus 
to the formation of protostars and starless cores in $\sim 0.02$ Myr. From the radio analysis (see Section 5.2), the lower limit for the dynamical age of this sub-region was calculated to be $\sim 0.11 \mathrm{Myr}$. The ages of Class 0 and Class I YSOs are generally of the order of a few 0.01 Myr and $0.1 \mathrm{Myr}$, respectively, and as such, their formation (and those of starless cores) could have been influenced by the expanding $\mathrm{H}$ II region. We add a caveat that though the numbers are consistent with a scenario where the high-mass star could have influenced the star formation at the periphery, this might not be the correct scenario necessarily owing to possible complex and hitherto unexamined morphological structures in this sub-region.

Based on the above discussions, the following appears to be a plausible chronology of star formation in this region. As the filaments formed and joined, they led to the formation of dense core at their junction, referred to as midriff in this paper. This seems to have initiated the formation of the central high-mass star(s) and other Class II/III sources. At this point, an external shock - as mentioned by (Rodney \& Reipurth 2008) - might have given a boost to the star formation going on. The highmass source IRS 1A South was formed at the junction, as well as the entire cluster which is centered upon this source. Due to lower density along the polar direction as opposed to the equatorial direction, when the high-mass star(s) formed, the Lyman continuum radiation escaped along the polar directions leading to the formation of the bipolar nebula. Fresh (spontaneous) star formation seems to be going on along the filamentary structures and their junction (albeit at lower stellar density) as evidenced by the younger sources in Class 0/I stages and starless cores. A few of BRCs and elephant-trunks exhibiting $\mathrm{H} \alpha$ emission to the south of the midriff might have undergone evolution under the influence of the ionizing region which has chiselled them out. The high-mass source IRS 5, which is distinct from the rest of the radio emission region, seems to have led to the formation of a separate arc-shaped nebula, and swept-up material to its periphery.

Future observations and analysis of the whole region in various molecular lines, radial velocity measurements, analysis of cluster properties using minimum-spanning tree method, understanding the mass and luminosity functions of the region, observations of indivdual BRCs, and spectral observations of other NIR bright sources will help in putting the star formation scenario on a firm footing.

\section{CONCLUSIONS}

In this paper, we carried out a multiwavelength study of the Galactic star-forming W40 H II region. Our main conclusions are as follows :

1. Using the MIR data from Spitzer in conjunction with NIR data from UKIRT, 1202 YSOs were identified in the region, out of which 40 are Class I sources and 1162 are Class II/III sources. Analysis of the YSO distribution and nearest-neighbour surface density yields the cluster radius as $\sim 0.44$ pc and peak surface density as $650 \mathrm{pc}^{-2}$. Mass calculation using extinction map yields a value of $\sim$ $126 \mathrm{M}_{\odot}$ within this radius.

2. The filamentary structures were examined to reveal 3 parsec scale filaments emanating from the midriff. Two of them (filaments ' 1 ' and ' 3 ' in our labelling) contain most of the youngest YSOs aligned along their lengths. Filament ' 2 ' was found to be relatively diffuse with hardly any of the youngest YSOs.

3. SED fitting using the radio continuum emission at $610 \mathrm{MHz}$ and $1280 \mathrm{MHz}$ for the total emission region yielded the value of electron density to be $\sim 1265 \pm 218 \mathrm{~cm}^{-3}$ and the total Lyman continuum luminosity to be $\sim 1.67 \times 10^{47}$ photons $\mathrm{s}^{-1}$. The dynamical age, for the ambient density ranging from 1000 to $15000 \mathrm{~cm}^{-3}$, ranges from $\sim 0.19$ to $0.78 \mathrm{Myr}$.

4. The IRS 5 arc-shaped nebular region was found to be distinct from the rest of the emission region, and was thus examined in radio separately. The electron density was obtained to be $288 \pm 55 \mathrm{~cm}^{-3}$ and a lower limit on the dynamical age to be $\sim 0.11$ Myr. A comparison of radio continuum photon luminosity with the tabulated values from Panagia (1973) shows IRS 5 to be of B1V spectral type, reaffirming previous estimate from Shuping et al. (2012). Extinction map gives a value of $\sim 71 \mathrm{M}_{\odot}$ as the mass for this arc-shaped nebula.

5. The star formation seems to have taken place in two successive epochs, with the formation of relativelyolder Class II/III and the central high-mass sources - resulting in a cluster which is centered around the high-mass star IRS 1A South - followed by that of the youngest sources (Class 0/I, and starless cores). A distinct case is of the IRS 5 nebular region, where material seems to have been swept-up to the edge of this arc-shaped nebula by the expanding $\mathrm{H}$ II region of the IRS 5 source.

We thank the anonymous referee for a thorough and critical reading of the manuscript, and for the suggestions which helped in improving this paper. K.K.M., M.S.N.K., D.K.O., and M.R.S. acknowledge support from Marie Curie IRSES grant (230843) under the auspices of which this work was carried out. K.K.M. would like to thank Hendrik Linz, MPIA (Heidelberg) for his invaluable help in obtaining the Herschel data. This research made use of data products from the Spitzer Space Telescope Archive. These data products are provided by the services of the Infrared Science Archive operated by the Infrared Processing and Analysis Centre/California Institute of Technology, funded by the National Aeronautics and Space Administration and the National Science Foundation.

\section{REFERENCES}

Allen, L., Bourke, T., Brooke, T., et al. 2006, Spitzer Proposal, 30574
Altenhoff, W. J., Downes, D., Goad, L., Maxwell, A., \& Rinehart, R. 1970, A\&AS, 1, 319 Alvarez, C., Feldt, M., Henning, T., et al. 2004, ApJS, 155, 123 
Arthur, S. J., Kurtz, S. E., Franco, J., \& Albarrán, M. Y. 2004, ApJ, 608, 282

Beaumont, C. N., \& Williams, J. P. 2010, ApJ, 709, 791

Bertin, E., \& Arnouts, S. 1996, A\&AS, 117, 393

Bessell, M. S., \& Brett, J. M. 1988, PASP, 100, 1134

Bohlin, R. C., Savage, B. D., \& Drake, J. F. 1978, ApJ, 224, 132

Bontemps, S., André, P., Könyves, V., et al. 2010, A\&A, 518, L85

Brand, J., Massi, F., Zavagno, A., Deharveng, L., \& Lefloch, B. 2011, A\&A, 527, A62

Cantalupo, C. M., Borrill, J. D., Jaffe, A. H., Kisner, T. S., \& Stompor, R. 2010, ApJS, 187, 212

Casali, M., Adamson, A., Alves de Oliveira, C., et al. 2007, A\&A, 467, 777

Casertano, S., \& Hut, P. 1985, ApJ, 298, 80

Chauhan, N., Pandey, A. K., Ogura, K., et al. 2009, MNRAS, 396, 964

Chavarría, L. A., Allen, L. E., Hora, J. L., Brunt, C. M., \& Fazio, G. G. 2008, ApJ, 682, 445

Ciardi, D. R., Woodward, C. E., Clemens, D. P., Harker, D. E., \& Rudy, R. J. 1998, A.J, 116, 349

Cohen, J. G., Persson, S. E., Elias, J. H., \& Frogel, J. A. 1981, ApJ, 249, 481

Dale, J. E., Ercolano, B., \& Bonnell, I. A. 2012, MNRAS, 424, 377

Davis, C. J., Kumar, M. S. N., Sandell, G., et al. 2007, MNRAS, 374,29

Dobashi, K., Uehara, H., Kandori, R., et al. 2005, PASJ, 57, 1

Evans, N. J., II, Allen, L. E., Blake, G. A., et al. 2003, PASP, 115, 965

Fich, M., \& Blitz, L. 1984, ApJ, 279, 125

Flaherty, K. M., Pipher, J. L., Megeath, S. T., et al. 2007, ApJ, 663,1069

Frerking, M. A., Langer, W. D., \& Wilson, R. W. 1982, ApJ, 262, 590

Goss, W. M., \& Shaver, P. A. 1970, Australian Journal of Physics Astrophysical Supplement, 14, 1

Gutermuth, R. A., Megeath, S. T., Myers, P. C., et al. 2009, ApJS, 184,18

Gutermuth, R. A., Megeath, S. T., Myers, P. C., et al. 2010, ApJS, 189,352

Hewett, P. C., Warren, S. J., Leggett, S. K., \& Hodgkin, S. T. 2006, MNRAS, 367, 454

Hillenbrand, L. A., Strom, S. E., Vrba, F. J., \& Keene, J. 1992 ApJ, 397, 613

Hodgkin, S. T., Irwin, M. J., Hewett, P. C., \& Warren, S. J. 2009 , MNRAS, 394, 675

Jenkins, E. B., \& Savage, B. D. 1974, ApJ, 187, 243

Kainulainen, J., Lehtinen, K., Väisänen, P., Bronfman, L., \& Knude, J. 2007, A\&A, 463, 1029

Könyves, V., André, P., Men'shchikov, A., et al. 2010, A\&A, 518, L106

Krumholz, M. R., Leroy, A. K., \& McKee, C. F. 2011, ApJ, 731, 25

Kuhn, M. A., Getman, K. V., Feigelson, E. D., et al. 2010, ApJ, 725,2485

Kurtz, S. E., Watson, A. M., Hofner, P., \& Otte, B. 1999, ApJ, 514,232

Lada, C. J. 1987, Star Forming Regions, 115, 1

Lada, C. J., \& Adams, F. C. 1992, ApJ, 393, 278

Lada, C. J., \& Lada, E. A. 2003, ARA\&A, 41, 57

Lada, C. J., Lada, E. A., Clemens, D. P., \& Bally, J. 1994, ApJ, 429,694

Lilley, A. E. 1955, ApJ, 121, 559

Martins, F., Schaerer, D., \& Hillier, D. J. 2005, A\&A, 436, 1049

Mathis, J. S. 1990, ARA\&A, 28, 37

Maury, A. J., André, P., Men'shchikov, A., Könyves, V., \& Bontemps, S. 2011, A\&A, 535, A77

Meyer, M. R., Calvet, N., \& Hillenbrand, L. A. 1997, AJ, 114, 288
Mezger, P. G., \& Henderson, A. P. 1967, ApJ, 147, 471

Mezger, P. G., Schraml, J., \& Terzian, Y. 1967, ApJ, 150, 807

Moran, J. M. 1983, Rev. Mexicana Astron. Astrofis., 7, 95

Myers, P. C. 2009, ApJ, 700, 1609

Nakamura, F., Sugitani, K., Shimajiri, Y., et al. 2011, ApJ, 737, 56

Ogura, K. 2010, Astronomical Society of India Conference Series, 1,19

Ojha, D. K., Tamura, M., Nakajima, Y., et al. 2004, ApJ, 608, 797

Ojha, D. K., Tamura, M., Nakajima, Y., et al. 2004, ApJ, 616, 1042

Panagia, N. 1973, AJ, 78, 929

Parker, Q. A., Phillipps, S., Pierce, M. J., et al. 2005, MNRAS, 362,689

Pilbratt, G. L., Riedinger, J. R., Passvogel, T., et al. 2010, A\&A, 518, L1

Pirogov, L., Zinchenko, I., Ojha, D. K., \& Ghosh, S. K. 2012, The Astronomer's Telegram, 4236, 1

Pirogov, L., Ojha, D. K., Thomasson, M., et al. 2013, MNRAS(accepted); arXiv:1309.6188

Radhakrishnan, V., Goss, W. M., Murray, J. D., \& Brooks, J. W. 1972, ApJS, 24, 49

Rieke, G. H., \& Lebofsky, M. J. 1985, ApJ, 288, 618

Rodney, S. A., \& Reipurth, B. 2008, Handbook of Star Forming Regions, Volume II, 683

Rodríguez, L. F., Rodney, S. A., \& Reipurth, B. 2010, AJ, 140, 968

Schmeja, S., Kumar, M. S. N., \& Ferreira, B. 2008, MNRAS, 389, 1209

Schmeja, S. 2011, Astronomische Nachrichten, 332, 172

Schneider, N., Csengeri, T., Hennemann, M., et al. 2012, A\&A, 540, L11

Shaver, P. A., \& Goss, W. M. 1970, Australian Journal of Physics Astrophysical Supplement, 14, 133

Shuping, R. Y., Snow, T. P., Crutcher, R., \& Lutz, B. L. 1999, ApJ, 520, 149

Shuping, R. Y., Vacca, W. D., Kassis, M., \& Yu, K. C. 2012, AJ, 144,116

Smith, J., Bentley, A., Castelaz, M., et al. 1985, ApJ, 291, 571

Spitzer, L. 1978, Physical Processes in the Interstellar Medium (New York: Wiley-Interscience)

Stahler, S. W., \& Palla, F. 2005, The Formation of Stars, by Steven W. Stahler, Francesco Palla, pp. 865. ISBN 3-527-405593. Wiley-VCH, January 2005.

Strömgren, B. 1939, ApJ, 89, 526

Swarup, G., Ananthakrishnan, S., Kapahi, V. K., et al. 1991, Current Science, Vol. 60, NO.2/JAN25, P. 95, 1991, 60, 95

Tenorio-Tagle, G. 1979, A\&A, 71, 59

Vallee, J. P. 1987, A\&A, 178, 237

Vallee, J. P., Guilloteau, S., \& MacLeod, J. M. 1992, A\&A, 266,

Westerhout, G. 1958, Bull. Astron. Inst. Netherlands, 14, 215

Ward-Thompson, D., \& Whitworth, A. P. 2011, An Introduction to Star Formation by Derek Ward-Thompson and Anthony P. Whitworth. Cambridge University Press, 2011. ISBN: 9780521630306

Waskett, T. J., Sibthorpe, B., Griffin, M. J., \& Chanial, P. F. 2007, MNRAS, 381, 1583

Whitworth, A. 1979, MNRAS, 186, 59

Quireza, C., Rood, R. T., Bania, T. M., Balser, D. S., \& Maciel, W. J. 2006, ApJ, 653, 1226

Yasui, C., Kobayashi, N., Tokunaga, A. T., Terada, H., \& Saito, M. 2008, ApJ, 675, 443

Zeilik, M., II, \& Lada, C. J. 1978, ApJ, 222, 896

Zhu, L., Wu, Y.-F., \& Wei, Y. 2006, Chinese J. Astron. Astrophys., 6,61 